OPEN ACCESS

Edited by:

Juan Pablo de Rivero Vaccari, University of Miami, United States

Reviewed by: Mariaelena Repici, University of Leicester, United Kingdom

Daniele Bottai,

University of Milan, Italy

*Correspondence: Gao Chen d-chengao@zju.edu.cn Yongjian Zhu

neurosurgery@zju.edu.cn

these authors have contributed equally to this work as co-first authors

¥These authors have contributed equally to this work as co-corresponding authors

Received: 05 October 2018 Accepted: 01 February 2019

Published: 22 February 2019

Citation:

Gao L, Zhang Z, Xu W, Li T, Ying G, Qin B, Li J, Zheng J, Zhao T,

Yan F, Zhu Y and Chen G (2019) Natrium Benzoate Alleviates Neuronal Apoptosis via the DJ-1-Related

Anti-oxidative Stress Pathway Involving Akt Phosphorylation in a Rat Model of Traumatic Spinal Cord Injury.

Front. Mol. Neurosci. 12:42. doi: 10.3389/fnmol.2019.00042

\section{Natrium Benzoate Alleviates Neuronal Apoptosis via the DJ-1-Related Anti-oxidative Stress Pathway Involving Akt Phosphorylation in a Rat Model of Traumatic Spinal Cord Injury}

Liansheng Gao't, Zhongyuan Zhang ${ }^{1 t}$, Weilin Xu1", Tao Li1, Guangyu Ying ${ }^{1}$, Bing Qin', Jianru Li' ${ }^{1}$, Jingwei Zheng ${ }^{1}$, Tengfei Zhao ${ }^{2}$, Feng Yan', Yongjian Zhu ${ }^{1 * \neq}$ and Gao Chen ${ }^{1 * \neq}$

${ }^{1}$ Department of Neurosurgery, Second Affiliated Hospital, School of Medicine, Zhejiang University, Hangzhou, China, ${ }^{2}$ Department of Orthopedics, Second Affiliated Hospital, School of Medicine, Zhejiang University, Hangzhou, China

This study aimed to explore the neuroprotective effects and mechanisms of natrium benzoate (NaB) and DJ-1 in attenuating reactive oxygen species (ROS)-induced neuronal apoptosis in traumatic spinal cord injury (t-SCl) in rats. T-SCl was induced by clip compression. The protein expression and neuronal apoptosis was evaluated by Western blotting, double immunofluorescence staining and transmission electron microscope (TEM). ROS level, spinal cord water content (SCWC) and Evans blue (EB) extravasation was also examined. Locomotor function was evaluated by Basso, Beattie, and Bresnahan (BBB) and inclined plane test (IPT) scores. We found that $\mathrm{DJ}-1$ is expressed in spinal cord neurons and increased after t-SCl. At $24 \mathrm{~h}$ postinjury, the levels of DJ-1, p-Akt, SOD2, ROS, p-p38 MAPK/p38 MAPK ratio, and CC-3 increased, while the Bcl-2/Bax ratio decreased. NaB upregulated DJ-1, p-Akt, and SOD2, decreased ROS, p-p38 MAPK/p38 MAPK ratio, and CC-3, and increased the $\mathrm{Bcl}-2 / \mathrm{Bax}$ ratio, which were reversed by DJ-1 siRNA. The proportion of CC-3and TUNEL-positive neurons also increased after t-SCl and was reduced by $\mathrm{NaB}$. These effects were reversed by MK2206. Moreover, the level of oxDJ-1 increased after t-SCl, which was decreased by DJ-1 siRNA, NaB or the combination of them. $\mathrm{NaB}$ also reduced mitochondrial vacuolization, SCWC and EB extravasation, and improved locomotor function assessed by the BBB and IPT scores. In conclusion, NaB increased DJ-1, and thus reduced ROS and ROS-induced neuronal apoptosis by promoting Akt phosphorylation in t-SCl rats. $\mathrm{NaB}$ shows potential as a therapeutic agent for t-SCl, with DJ-1 as its main target.

Keywords: natrium benzoate, DJ-1, oxidative stress, reactive oxygen species, apoptosis, traumatic spinal cord injury

Abbreviations: AD, Alzheimer's disease; ANOVA, analysis of variance; BBB, Basso, Beattie, and Bresnahan; BSCB, bloodspinal cord barrier; CC-3, cleaved caspase-3; CNS, central nervous system; EB, Evans Blue; IF, immunofluorescence; IPT, inclined plane test; i.t., intrathecal injection; MS, multiple sclerosis; NaB, natrium benzoate; oxDJ-1, oxidized DJ-1; PD, Parkinson's disease; ROS, reactive oxygen species; SCWC, spinal cord water content; SD, Sprague-Dawley; siRNA, small interfering RNA; SOD, superoxide dismutase; TEM, transmission electron microscopy; t-SCI, traumatic spinal cord injury; TUNEL, terminal deoxynucleotidyl transferase-mediated dUTP nick end labeling. 


\section{INTRODUCTION}

Traumatic spinal cord injury is one of the most serious injuries among all traumas worldwide and generally results in severe and permanent neurological dysfunction and neurodegeneration (Seo et al., 2015). The pathophysiology of t-SCI involves a primary injury followed by secondary damage. Following trauma, the primary injury occurs immediately, which comprises of a tissue contusion, axonal fracture, and a vascular rupture. The secondary injury results from diverse molecular, cellular, and biochemical responses induced by the primary injury and may persist for hours, days, or weeks; these responses include the oxidative stress reaction (Lam et al., 2013; Fatima et al., 2015).

During oxidative stress, molecular oxygen is inadequately reduced in the mitochondria, resulting in excessive levels of ROS. Under normal conditions, a balance between ROS generation and degradation is maintained (Droge, 2002; Valko et al., 2007). However, the ROS level is significantly increased in the traumatic spinal cord (Ahuja et al., 2017). Excessive ROS leads to various types of destructive effects such as lipid peroxidation, protein oxidation, and DNA damage. Additionally, ROS can activate specific cascades that induce cell death (Sugawara and Chan, 2003). It was reported that ROS can induce cell apoptosis by activating the p38 MAPK signaling pathway (Wang et al., 2003; Huang et al., 2018), which plays an important role in the pathogenesis of t-SCI. Neuronal apoptosis is one of the many factors contributing to the poor prognosis of t-SCI (Niu and Yip, 2011). Thus, reducing oxidative stressinduced neuronal apoptosis following t-SCI, may have important therapeutic effects.

Natrium benzoate, the sodium salt of an aromatic carboxylic acid, is a metabolite of cinnamon. It is commonly used as a flavoring material and as a preservative for a variety of foods and cosmetics (Nair, 2001) and has a long history of medical use. Williams and Lock (2005) reported that $\mathrm{NaB}$ attenuated D-serineinduced nephrotoxicity in rats. It was previously demonstrated that treatment with $\mathrm{NaB}$ upregulated $\mathrm{T}_{\text {reg }}$ cells and ameliorated relapsing-remitting experimental allergic encephalomyelitis in MS mice (Brahmachari and Pahan, 2007). NaB was also used as a drug to treat hyperammonemia caused by hepatic metabolic defects, such as urea cycle defects in children (approved by the US FDA) (Scaglia et al., 2004; Gropman et al., 2007; Misel et al., 2013). In the CNS, $\mathrm{NaB}$ can inhibit activated glial cells to express a variety of proinflammatory factors (Brahmachari et al., 2009). Modi et al. (2015) found that $\mathrm{NaB}$ decreased the generation of ROS in activated microglia. Jana et al. (2013) reported that $\mathrm{NaB}$ increased the expression of neurotrophic factors (BDNF and NT3) via the PKA-CREB pathway both in vivo and in vitro. Recently, an increasing number of studies have demonstrated that $\mathrm{NaB}$ has neuroprotective effects in $\mathrm{AD}, \mathrm{PD}$, and other neurodegenerative disorders by upregulating the expression of DJ-1 (Khasnavis and Pahan, 2012, 2014). However, the potential mechanisms remain unclear.

DJ-1, also known as PARK7, is a highly conserved protein expressed in the tissues of nearly all organisms ranging from bacteria to humans (Bonifati et al., 2003). The DJ-1 gene was originally identified as an oncogene and mutations in this gene were found to be responsible for familial PD (Nagakubo et al., 1997). In the CNS, DJ-1 is expressed in neurons, astrocytes, and microglia (Bandopadhyay et al., 2004; Kim et al., 2013). DJ-1 mainly localizes in the cytoplasm and a small amount is found in the nucleus and mitochondria. The correlation between its subcellular distribution and biological function remains unclear (Junn et al., 2009). DJ-1 has diverse functions and is involved in multiple physiological activities, such as oncogenesis (Nagakubo et al., 1997), protein-RNA interactions (Hod et al., 1999; Van Der Brug et al., 2008; Blackinton et al., 2009a), transcriptional regulation (Xu et al., 2005; Zhong et al., 2006; Takahashi-Niki et al., 2017), molecule chaperone (Shendelman et al., 2004; Zhou et al., 2006; Batelli et al., 2008), fertilization (Okada et al., 2002; Yoshida et al., 2003), mitochondrial function regulation (Shimizu et al., 2016), glycation damage prevention (Advedissian et al., 2016), and, most importantly, the oxidative stress reaction (Pantcheva et al., 2014). DJ-1 has shown neuroprotective effects in neurodegenerative diseases and ischemic stroke. Injection of DJ-1 into the substantia nigra reduced neuronal death and improved motor functions in a rat model of PD (Inden et al., 2006). DJ-1 protected against ischemia and reperfusion damage in focal cerebral ischemia rats (Yanagisawa et al., 2008; Yanagida et al., 2009). The loss of DJ1 aggravated neuronal impairment caused by cerebral ischemia (Aleyasin et al., 2007).

The potential neuroprotective mechanism of DJ-1 may rely on its ability to rescue cells from oxidative stress (Pantcheva et al., 2014). Several studies have clarified the important functions of DJ-1, including oxidative stress sensing and ROS scavenging in the brain (Taira et al., 2004; Zhang et al., 2005). It has been reported that DJ-1 is sensitive to oxidation and its protein level is upregulated by oxidative stress induction (Mitsumoto et al., 2001; Jin et al., 2005). DJ-1 knockdown cells or DJ-1 knockout mice were prone to suffering cell death after treatment with $\mathrm{H}_{2} \mathrm{O}_{2}$ or other neurotoxins (Yokota et al., 2003; Martinat et al., 2004; Chen et al., 2005; Kim et al., 2005b). The exact mechanisms of how DJ-1 protects against oxidative stress remain unclear, but it may enhance the phosphorylation of Akt (Kim et al., 2005a; Yang et al., 2005; Di Segni et al., 2006; Gorner et al., 2007; Van Der Brug et al., 2008; Aleyasin et al., 2010; Wang et al., 2014; Zhang et al., 2016). However, whether this capacity of DJ-1 functions in $\mathrm{t}-\mathrm{SCI}$ is unknown.

DJ-1 is a potential target for treating various neurodegenerative diseases and ischemic stroke. However, the effects of DJ-1 on t-SCI and its possible mechanisms have not been investigated. In the current study, we confirmed that $\mathrm{NaB}$ treatment reduces oxidative stress-induced neuronal apoptosis, thus promoting functional recovery after t-SCI in rats, possibly via DJ-1-mediated Akt phosphorylation.

\section{MATERIALS AND METHODS}

\section{Animal Model}

Adult male SD rats $(250-300 \mathrm{~g})$ were obtained from the Slac Laboratory Animal Co., Ltd. (Shanghai, China). All rats were reared in an environment of constant temperature and 
humidity and with a normal circadian rhythm. The rats were intraperitoneally injected with $1 \%$ pentobarbital $(40 \mathrm{mg} / \mathrm{kg})$ before surgery. A $2-\mathrm{cm}$ midline incision was made at the level of the T10 vertebra. After exposure of the lamina, laminectomy of T10 was performed to reveal the spinal cord. Next, a vascular clip (30 g force, INS 14120, Kent Scientific, Torrington, CT, United States) was used to clamp the spinal cord for $30 \mathrm{~s}$ without destroying the dura mater, which would cause a spinal cord compression injury (Liang et al., 2010). The sham rats were subjected to a similar surgery but without the clamp. Each rat was intraperitoneally injected with $5 \mathrm{~mL}$ saline for a water complement after surgery and was kept warm during recovery. Bladder massages were performed twice daily to facilitate urination until the recovery of urinary function.

\section{Drugs and Small Interfering RNA (siRNA)}

Natrium benzoate $(100 \mathrm{mg} / \mathrm{kg}$, Sigma-Aldrich, St. Louis, MO, United States) was dissolved in $100 \mu \mathrm{L}$ of water and the rats were treated with $\mathrm{NaB}$-containing water via gavage at $1 \mathrm{~h}$ after t-SCI (Khasnavis and Pahan, 2014; Kundu et al., 2016). An Akt inhibitor, MK2206, (100 $\mu \mathrm{g}$, Selleck Chemicals, Houston, TX, United States) was dissolved in dimethyl sulfoxide and further diluted in $10 \mu \mathrm{L}$ of sterile saline. The rats were treated with MK2206-containing normal saline via intrathecal injections at $1 \mathrm{~h}$ after t-SCI (Yan et al., 2017).

Two target-specific siRNAs disturbing rat DJ-1 mRNA mixtures (sense: 5'-CCCAUUGGCUAAGGACAAATT-3', 5'UGGAGACGGUCAUCCCUGUTT-3') or scramble siRNA (sense: 5'-UUCUCCGAACGUGUCACGUTT-3') obtained from Thermo Fisher Scientific (Waltham, MA, United States) were dissolved in Entranster ${ }^{\mathrm{TM}}$-in vivo transfection reagent (500 pmol/10 $\mu \mathrm{L}$, Engreen Biosystem, Beijing, China). The rats were intrathecally injected with siRNA solution at $48 \mathrm{~h}$ before t-SCI as previously described (Figueroa et al., 2016).

\section{Intrathecal (i.t.) Injection}

Intrathecal injections were administered as previously described (Hylden and Wilcox, 1980). Briefly, the rat was fixed in one hand with its back arched, while the other hand held a syringe positioned at $20^{\circ}$ over the spine with its needle tip pointing forward to puncture the subarachnoid space via the intervertebral space between L5 and L6. The injection speed was $2 \mu \mathrm{L} / \mathrm{min}$. After injection, the needle was kept in situ for an additional 10 min before seceding. The sham rats were subjected to the same puncture but without drug injection.

\section{Study Design \\ Experiment 1}

We randomly allocated the rats into seven groups: $\operatorname{sham}(N=12)$, t-SCI $3 \mathrm{~h}(N=6)$, t-SCI $6 \mathrm{~h}(N=6)$, t-SCI $12 \mathrm{~h}(N=6)$, t-SCI $24 \mathrm{~h}(N=12)$, t-SCI $48 \mathrm{~h}(N=6)$, and t-SCI $72 \mathrm{~h}(N=6)$. Six rats in each group were used to detect the changes in DJ-1 and p-Akt expression over time by Western blotting. Six rats in the sham and t-SCI $24 \mathrm{~h}$ groups were used for double IF staining of DJ-1 and NeuN.

\section{Experiment 2}

To investigate the functions of DJ-1, we randomly distributed the rats into six groups: sham $(N=24)$, t-SCI + vehicle $(N=24)$, t-SCI + scramble siRNA $(N=6)$; t-SCI + DJ-1 siRNA $(N=6)$, $\mathrm{t}-\mathrm{SCI}+\mathrm{NaB}(N=24)$, and $\mathrm{t}-\mathrm{SCI}+\mathrm{NaB}+\mathrm{DJ}-1$ siRNA $(N=6)$. At $24 \mathrm{~h}$ post-injury, six rats from each group were used to quantify the levels of DJ-1, oxDJ-1, Akt, SOD2, p38 MAPK, Bcl2 , Bax, and CC-3 by Western blotting. ROS were measured in the other six rats in these groups. EB extravasation and SCWC were detected using the other six rats in the sham, t-SCI + vehicle, and t-SCI $+\mathrm{NaB}$ groups, respectively. Another six rats in these groups were used to observe the ultrastructure of the cells by TEM.

\section{Experiment 3}

To examine the long-term functions of DJ-1 in neurological improvement, we randomly allotted the rats into three groups: sham $(N=6)$, t-SCI + vehicle $(N=6)$, and t-SCI $+\mathrm{NaB}(N=6)$. All rats were treated for seven consecutive days post-injury. The BBB and IPT scores were determined before and at 1, 3, 7, 14, 21, and 28 days after treatment in all groups.

\section{Experiment 4}

To analyze the mechanism of action of DJ-1, we randomly assigned the rats into five groups: sham $(N=6)$, t-SCI + vehicle $(N=18), \mathrm{t}-\mathrm{SCI}+\mathrm{NaB}(N=18), \mathrm{t}-\mathrm{SCI}+\mathrm{MK} 2206(N=18)$, and $\mathrm{t}-\mathrm{SCI}+\mathrm{NaB}+\mathrm{MK} 2206(N=18)$. At $24 \mathrm{~h}$ post-injury, six rats in each group, except the sham group, were used to quantify the expression levels of DJ-1, Akt, SOD2, p38 MAPK, Bcl-2, Bax, and CC-3 by Western blotting. ROS levels were measured in the other six rats in these groups. Another six rats in each group were used for TUNEL, CC-3, and NeuN double IF staining.

The detailed experimental design is shown in Figure 1.

\section{Motor Function Assessment}

The locomotor functions of rats in each group were evaluated by determining the BBB (Basso et al., 1995) and IPT (Rivlin and Tator, 1977) scores on days 1, 3, 7, 14, 21, and 28 after t-SCI. The detailed BBB scores are shown in Table 1. IPT was conducted as follows. The long axis of the rat body and long axis of the inclined plate were placed vertically. Gradually, the angle between the inclined plate and horizontal plane was increased until the rats could just remain on the inclined plate for $5 \mathrm{~s}$, and this angle was recorded. The researcher was blinded to the rat group assignments during evaluation. Each rat was assessed three times and the average value was taken as the final score.

\section{SCWC Examination}

The SCWC was detected using the wet-dry method ( $\mathrm{Hu}$ et al., 2015). Spinal cord samples of $1.5-\mathrm{cm}$ long, where the injured site was centered, were obtained. The samples were weighted immediately (wet weight). Next, they were dried at $95^{\circ} \mathrm{C}$ for $72 \mathrm{~h}$ and then weighted (dry weight). The researcher was blinded to the rat group assignments. The results were presented as the water content (g) in unit wet tissue (g). 
Exp. 1

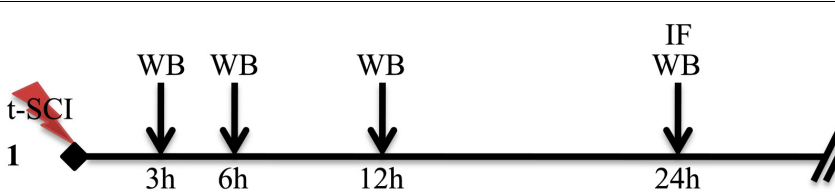

Group: Sham, t-SCI 3h, t-SCI 6h, t-SCI 12h, t-SCI 24h, t-SCI 48h, t-SCI $72 \mathrm{~h}$.

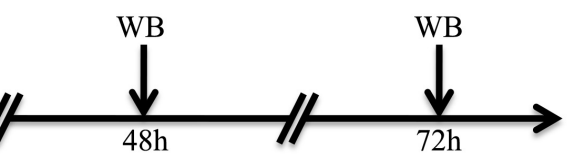

DJ-1 siRNA or scramble siRNA (i.t.)

Exp. 2

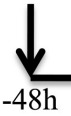

$\mathrm{NaB}$ (gavage)

ROS
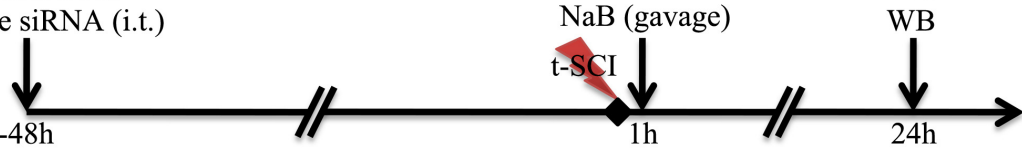

Group: Sham, t-SCI+vehicle, t-SCI+scramble siRNA, t-SCI+DJ-1 siRNA, t-SCI+NaB, t-SCI+NaB+DJ-1 siRNA.

SCWC

$\mathrm{NaB}$ (gavage)

EB

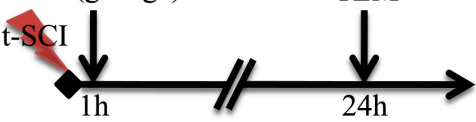

$\mathrm{NaB}$ (gavage, d0-d6)

Group: Sham, t-SCI+vehicle, t-SCI+NaB.

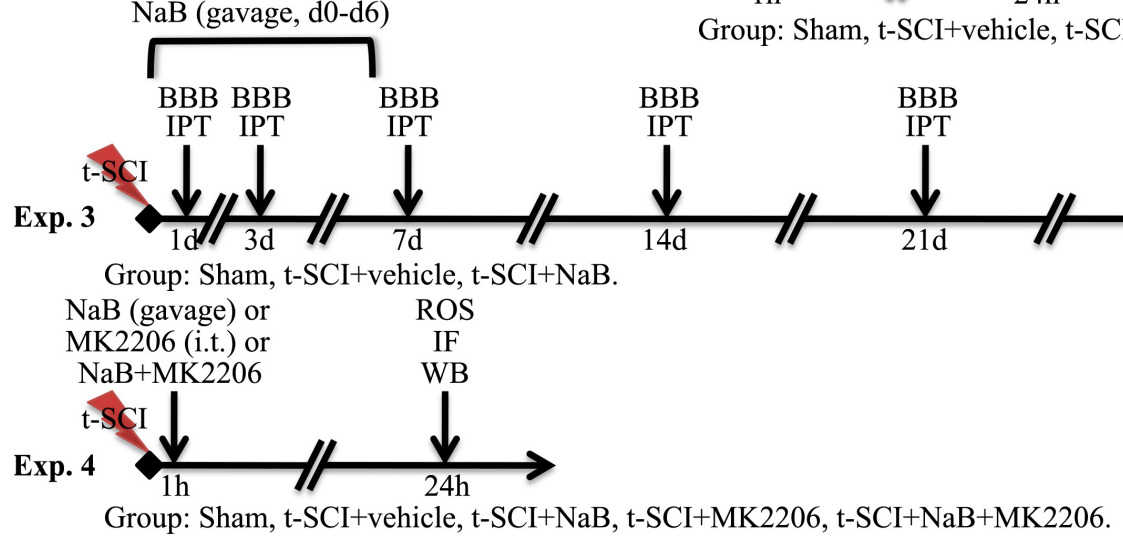

FIGURE 1 | Detailed experimental design.

\section{Blood-Spinal Cord Barrier (BSCB) Leakage}

Blood-spinal cord barrier leakage was assessed by EB extravasation (Li et al., 2014; Zhou et al., 2017). EB solution (3\%, $45 \mathrm{mg} / \mathrm{kg}$, Sigma-Aldrich) was administrated through the femoral vein after anesthetization. One hour later, spinal cord samples of $1.0-\mathrm{cm}$ long, where the injured site was centered, were obtained after transcardial perfusion with $0.1 \mathrm{M}$ PBS. The sample was homogenized in $3 \mathrm{~mL} \mathrm{50 \%} \mathrm{trichloroacetic} \mathrm{acid} \mathrm{and} \mathrm{centrifuged} \mathrm{at}$ $12,000 \times g$ at $4^{\circ} \mathrm{C}$ for $20 \mathrm{~min}$. Next, $1 \mathrm{~mL}$ of the supernatant was mixed with $1 \mathrm{~mL}$ of ethanol and trichloroacetic acid solution (1:3) and incubated at $4^{\circ} \mathrm{C}$ overnight. The sample was then centrifuged at $15,000 \times g$ at $4^{\circ} \mathrm{C}$ for $30 \mathrm{~min}$. The supernatant was examined at an emission wavelength of $680 \mathrm{~nm}$ and excitation wavelength of $620 \mathrm{~nm}$ with a microplate reader (Bio-Tek Instruments, Winooski, VT, United States). The fluorescence value was converted to an EB content ( $\mu \mathrm{g}$ ) according to the standard curve. The researcher was blinded to the group assignments of the rats. Each test was conducted twice, and the average value was taken as the result. The results were expressed as the EB content ( $\mu \mathrm{g})$ in unit tissue (g).

\section{Western Blot Analysis}

Spinal cord samples of $0.5-\mathrm{cm}$ long, where the injured site was centered, were obtained after transcardial perfusion with $0.1 \mathrm{M}$ PBS. The tissues were homogenized in RIPA lysis buffer containing protease inhibitors (1\%) and phosphates inhibitors (1\%), and then centrifuged at $12,000 \times g$ at $4^{\circ} \mathrm{C}$ for $15 \mathrm{~min}$. The protein concentration of the supernatant was measured using a BCA protein assay kit (Pierce, Rockford, IL, United States). Each sample was diluted to the same protein concentration $(4 \mu \mathrm{g} / \mu \mathrm{L})$ with RIPA and resuspended in loading buffer. An equal volume of protein samples $(10 \mu \mathrm{L})$ was loaded into each well of a $12 \%$ SDSPAGE gel. The proteins were separated by electrophoresis and then transferred onto a polyvinylidene fluoride membrane (BioRad Laboratories, Hercules, CA, United States). The membrane was blocked in $10 \%$ skim milk for $1 \mathrm{~h}$. After rinsing with Tris-buffered saline and Tween 20, specific primary antibodies including anti-DJ-1 (1:20,000, ab76008, Abcam, Cambridge, United Kingdom), anti-oxDJ-1 (1:5000, ab169520, Abcam), anti-p-Akt (1:2000, CST\#4060s, Cell Signaling Technology, Danvers, MA, United States), anti-Akt (1:1000, CST\#9272s, Cell Signaling Technology), anti-SOD2 (1:5000, ab13533, Abcam), 
TABLE 1 | BBB score.

\begin{tabular}{|c|c|}
\hline Score & Description \\
\hline 0 & No hind limb movement. \\
\hline 1 & Slight movement of one or two joints, typically hip and/or knee joints. \\
\hline 2 & One joint moves extensively, or one joint moves extensively and another joint moves slightly. \\
\hline 3 & Extensive movement of both joints. \\
\hline 4 & All three joints of the hind limb can move slightly. \\
\hline 5 & Two joints move slightly, and the third can move extensively. \\
\hline 6 & Two joints move extensively, and the third joint can move slightly. \\
\hline 7 & All three joints of the hind limb can move extensively. \\
\hline 8 & The palm can touch the ground under non-load-bearing conditions. \\
\hline 9 & Occasionally, the palm can support or back can move under load-bearing conditions, no movement under palm support. \\
\hline 10 & Occasionally, the palm moves under load-bearing conditions, and there is no coordinated movement of fore-hind limbs. \\
\hline 11 & More palm load-bearing movement, but no coordinated movement of fore-hind limbs. \\
\hline 12 & More palm load-bearing movement, occasional coordinated movement of fore-hind limbs. \\
\hline 13 & Common palm load-bearing movement, common coordinated movement of fore-hind limb. \\
\hline 14 & $\begin{array}{l}\text { Persistent palm load-bearing movement and coordinated movement of fore-hind limb; or common palm movement and persistent coordinated } \\
\text { movement of fore-hind limb, occasional back movement. }\end{array}$ \\
\hline 15 & $\begin{array}{l}\text { Persistent palm movement and coordinated movement of fore-hind limbs, no or occasional grip during fore limb advancement; the position of active claw } \\
\text { is parallel to the body at initial contact. }\end{array}$ \\
\hline 16 & $\begin{array}{l}\text { Persistent palm movement and coordinated movement of fore-hind limbs, common grip during fore limb advancement; the position of active claw is } \\
\text { parallel to the body at initial contact and rotated after weight transfer. }\end{array}$ \\
\hline 17 & $\begin{array}{l}\text { Persistent palm movement and coordinated movement of fore-hind limbs, common grip during fore limb advancement; the position of active claw is } \\
\text { parallel to the body at initial contact and after weight transfer. }\end{array}$ \\
\hline 18 & $\begin{array}{l}\text { Persistent palm movement and coordinated movement of fore-hind limbs, persistent grip during fore limb advancement; the position of active claw is } \\
\text { parallel to the body at initial contact and rotated after weight transfer. }\end{array}$ \\
\hline 19 & $\begin{array}{l}\text { Persistent palm movement and coordinated movement of fore-hind limbs, persistent grip during fore limb advancement; the position of active claw is } \\
\text { parallel to the body at initial contact and after weight transfer. The tail sometimes or always droops. }\end{array}$ \\
\hline 20 & $\begin{array}{l}\text { Persistent palm movement, coordinated gait, toe grip, the position of active claw is parallel to the body at initial contact and after weight transfer. The } \\
\text { trunk is unstable, and the tail keeps tilting. }\end{array}$ \\
\hline 21 & Persistent palm movement, coordinated gait, toe grip, the position of active claw is always parallel to the body, the trunk is stable, and the tail keeps tilting. \\
\hline
\end{tabular}

TABLE 2 | Main physiological data for rats in each group.

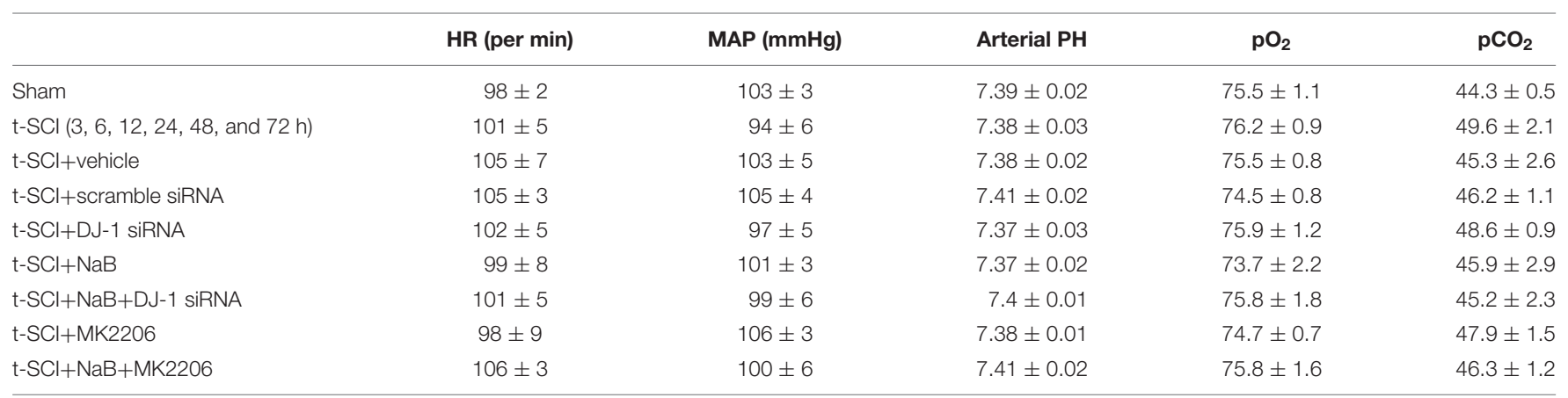

siRNA, small interfering RNA; NaB, natrium benzoate; $t$-SCl, traumatic spinal cord injury; HR, heart rate; MAP, mean arterial pressure.

anti-p-p38 MAPK (1:1000, ab4822, Abcam), anti-p38 MAPK (1:1000, ab31828, Abcam), anti-Bax (1:1000, ab32503, Abcam), anti-Bcl-2 (1:500, ab59348, Abcam), anti-CC-3 (1:500, ab13847, Abcam), and anti- $\beta$-actin (1:5000, ab8226, Abcam) were incubated with the membranes at $4^{\circ} \mathrm{C}$ overnight. After rinsing, the membranes were treated with appropriate secondary antibodies (1:10,000, ZB-2301 or ZB-2305, Zhongshan Golden Bridge, Beijing, China) for $1 \mathrm{~h}$ at $25^{\circ} \mathrm{C}$ and rinsed again. The membranes were immersed in a mixture of equal amounts of the A and B solutions from an ECL kit (Immobilon, Millipore, Billerica, MA, United States) and then observed using a chemical imager (Bio-Rad Laboratories) to detect the proteins.

The gray level of each band was analyzed using Image software (National Institutes of Health, Bethesda, MD, United States). The results were displayed as the ratios of gray levels of the protein of interest to the internal control proteins and normalized against the sham group.

\section{IF and TUNEL Dyeing}

Spinal cord samples of $1.0-\mathrm{cm}$ long, where the injured site was centered, were obtained after transcardial perfusion with 
$0.1 \mathrm{M}$ PBS. Following fixation in $4 \%$ paraformaldehyde at $4^{\circ} \mathrm{C}$ for $24 \mathrm{~h}$ and dehydration in $30 \%$ sucrose solution at $4^{\circ} \mathrm{C}$ for $72 \mathrm{~h}$, three axial frozen sections $(20 \mu \mathrm{m})$ were obtained from the proximal spinal cord, while another three sections were obtained from the distal spinal cord $2 \mathrm{~mm}$ away from the center of the injury site, respectively, giving a total of six sections from each sample (Kjell et al., 2016; Wang et al., 2017). After disrupting the cell membranes with $0.3 \%$ Triton $X-$ 100 for $15 \mathrm{~min}$, blocking with $10 \%$ donkey serum for $2 \mathrm{~h}$ at $25^{\circ} \mathrm{C}$, and rinsing with $0.01 \mathrm{M}$ PBS, specific primary antibodies including anti-DJ-1 (1:500, ab76008, Abcam), anti-CC-3 (1:200, ab13847, Abcam), and anti-NeuN (1:500, ab104224, Abcam) were incubated with the sections at $4^{\circ} \mathrm{C}$ overnight. The sections were incubated for $2 \mathrm{~h}$ at $25^{\circ} \mathrm{C}$ with appropriate secondary antibodies (1:500, Invitrogen, Thermo Fisher Scientific) or TUNEL dye liquor (Roche, Basel, Switzerland). DAPI (1 $\mu \mathrm{g} / \mathrm{mL}$, SigmaAldrich) was used to stain the nucleus, and then the sections were mounted. Finally, the sections were observed with a fluorescence microscope (Olympus, Tokyo, Japan) and the photos were processed with Photoshop 13.0 software (Adobe Systems, Inc., San Jose, CA, United States).

One random grey matter field per section was used to count the number of cells in the photos at $200 \times$ magnification. The expression of DJ-1 was evaluated as the mean ratio of DJ1 -positive neurons to total neurons in each group. Neuronal apoptosis was evaluated as the mean ratio of CC-3-/TUNELpositive neurons to total neurons in each group.

\section{Measurement of ROS Levels}

The ROS levels in each group were tested with an ROS assay kit (Jiancheng, Nanjing, China) according to the manufacturer's instructions. Spinal cord samples of $0.5-\mathrm{cm}$ long, where the injured site was centered, were obtained after transcardial perfusion with $0.1 \mathrm{M}$ PBS. After homogenization in $0.01 \mathrm{M}$ PBS and centrifugation at $1,000 \times g$ at $4^{\circ} \mathrm{C}$ for $10 \mathrm{~min}$, the supernatant from each sample $(190 \mu \mathrm{L})$ and DCFH-DA $(10 \mu \mathrm{L}$, $1 \mathrm{~mol} / \mathrm{L}$ ) were mixed in a 96 -well plate at $37^{\circ} \mathrm{C}$ for $30 \mathrm{~min}$ and then examined at an emission wavelength of $525 \mathrm{~nm}$ and
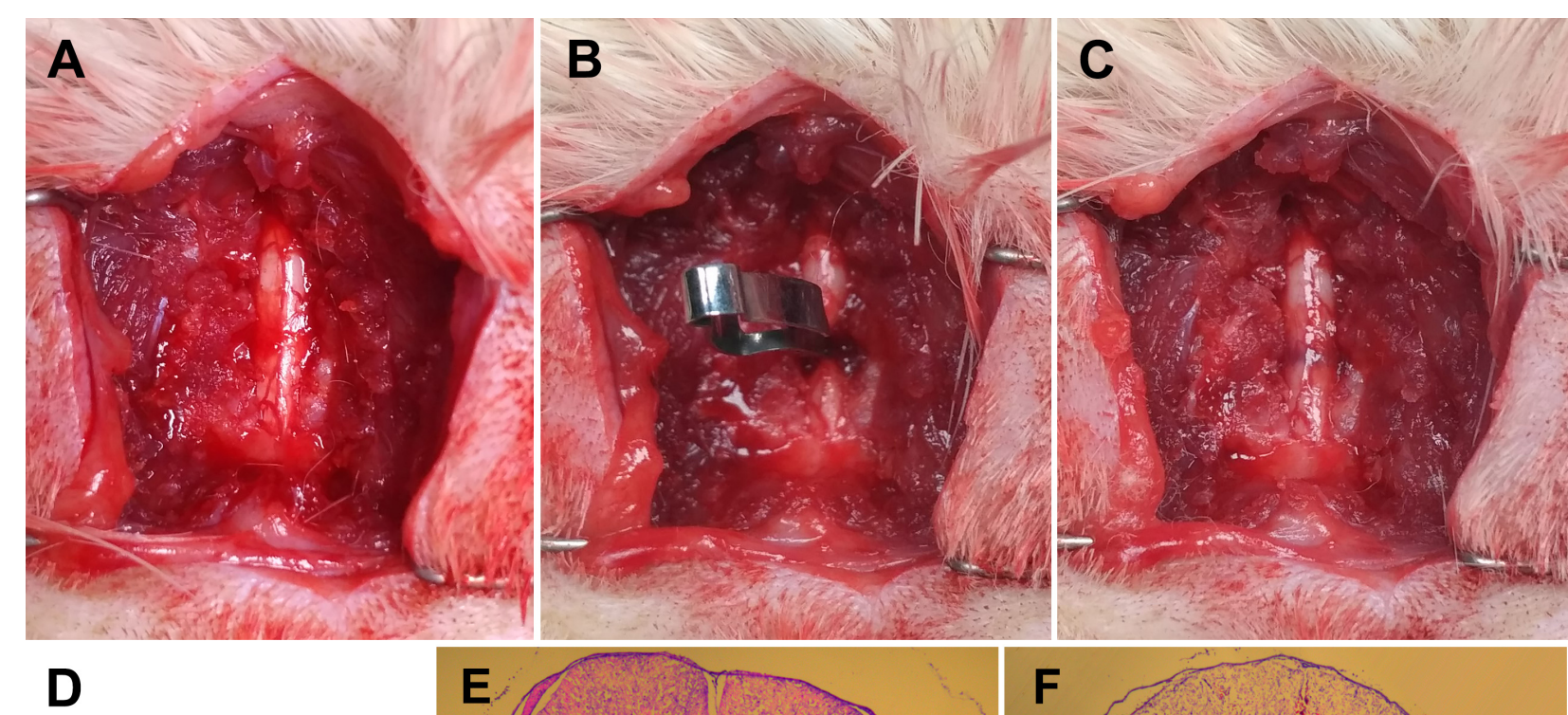
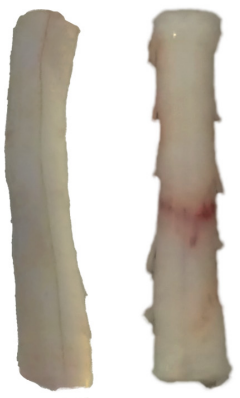

a
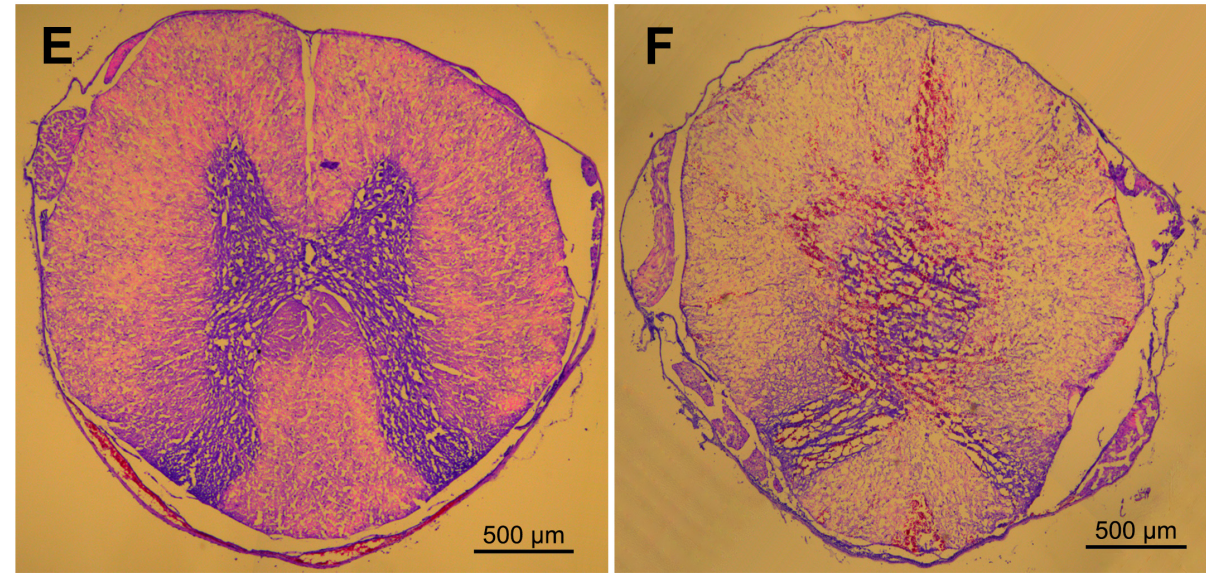

FIGURE 2 | Representative figures showing the surgical procedures used to establish the clip compressive model of t-SCl: the exposed rat spinal cord (A); compression of the exposed spinal cord with a vascular clip (B); crushed site (C). Representative spinal cord samples showing normal spinal cord (D, a) and crushed injury of spinal cord by vascular clip (D, b). Typical axial spinal cord sections from the spinal cord: normal spinal cord structure in the sham group (E); spinal cord swelling, disorganization, and bleeding in the t-SCl 24 h group (F). 
excitation wavelength of $500 \mathrm{~nm}$ with a microplate reader (BioTek Instruments, Inc.). Additionally, the protein concentration of the supernatant was tested using a BCA protein assay kit (Pierce). The researcher was blinded to the assignments of the rats in each group. Each test was conducted twice, and the average value was taken as the result. The ROS levels were displayed as fluorescence/mg protein.

\section{TEM}

Spinal cord samples of $0.5-\mathrm{cm}$ long, where the injured site was centered, were obtained after transcardial perfusion with $0.1 \mathrm{M}$
PBS. Two 1- $\mathrm{mm}^{3}$ tissue blocks were acquired from the gray matter regions of the proximal and distal spinal cord $2 \mathrm{~mm}$ away from the center of the injury site, respectively, and then incubated in $2.5 \%$ glutaraldehyde for fixation overnight. The tissue blocks were subjected to rinsing, fixation in $1 \%$ osmic acid for $1 \mathrm{~h}$, gradient alcohol dehydration, and embedment in araldite at $60^{\circ} \mathrm{C}$ overnight. Three 100-nm ultra-thin sections were obtained from each block, giving a total of six sections from each sample. A TEM (Philips Tecnai 10, Amsterdam, Netherlands) was applied to observe the slices, which had been stained with uranyl acetate and lead citrate.
A
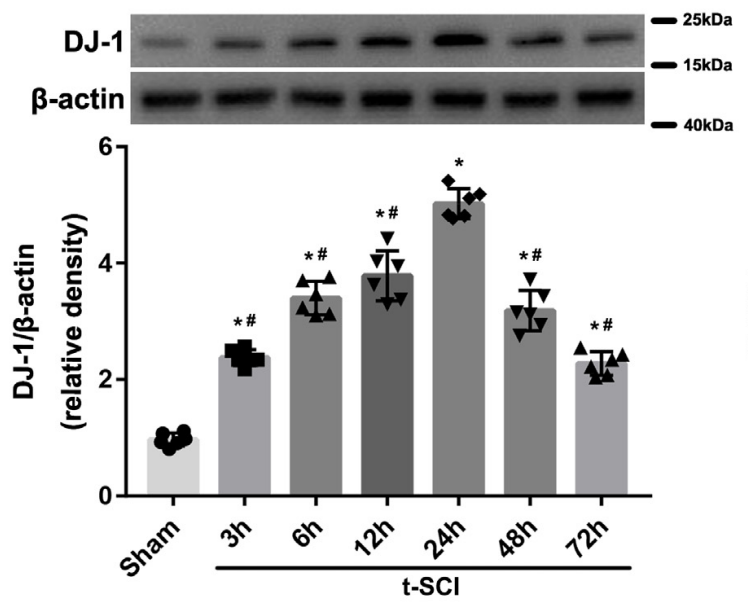

C
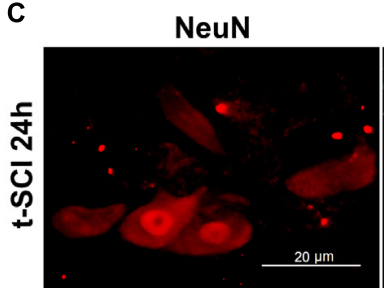

D
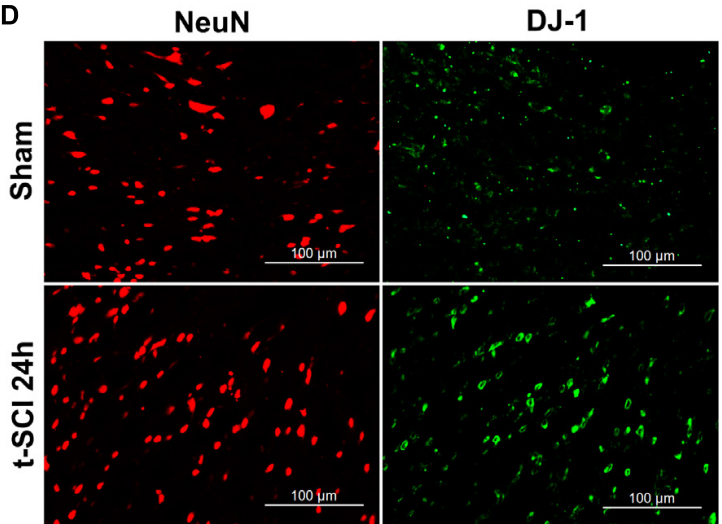

DJ-1

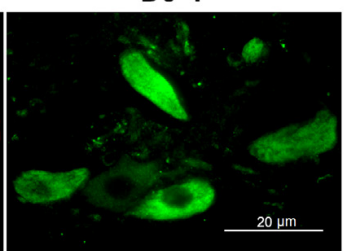

DJ-1

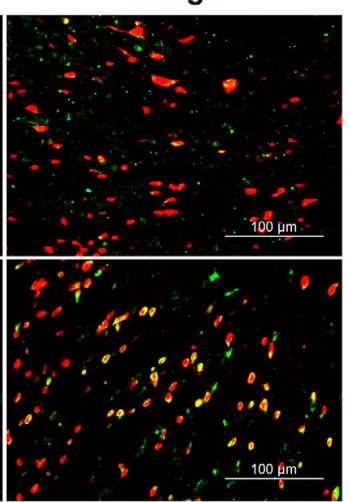

B
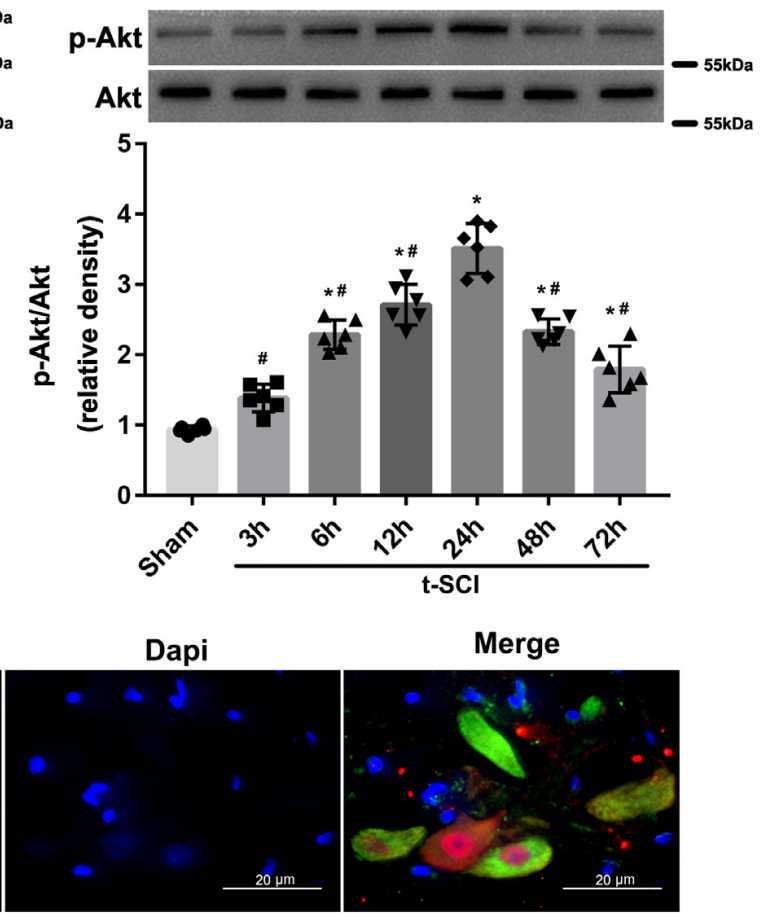

Merge
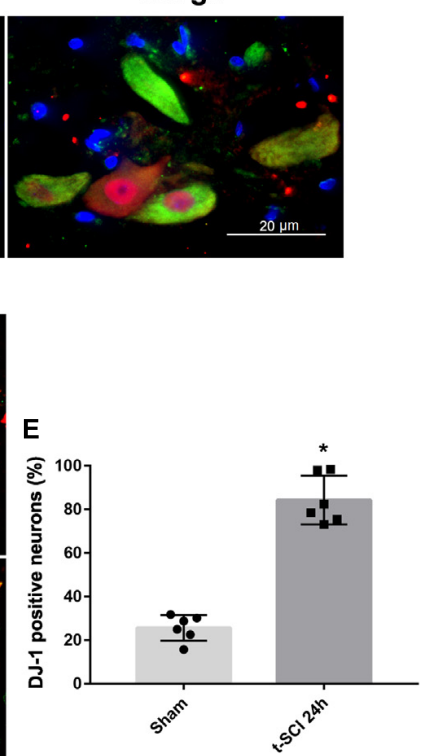

FIGURE 3 | Representative Western blots showing the time course of changes in the levels of DJ-1 and p-Akt. The expression level of DJ-1 was significantly elevated at $3 \mathrm{~h}$ and peaked at $24 \mathrm{~h}$ after t-SCl, and then significantly decreased after $24 \mathrm{~h}$ post-injury (A); the expression level of p-Akt was significantly increased at $6 \mathrm{~h}$ and peaked at $24 \mathrm{~h}$ after t-SCl, and then significantly decreased after $24 \mathrm{~h}$ post-injury (B). $\mathrm{N}=6$ for each group. Data is expressed as mean $\pm \mathrm{SD}$ and analyzed by one-way ANOVA and Bonferroni's post hoc multiple comparisons test. Representative IF staining micrographs showing the proportion of DJ-1-positive neurons in each group at $24 \mathrm{~h}$ post-injury. DJ-1 was expressed mainly in neurons (C) and proportion of DJ-1-positive neurons was increased after t-SCl (D,E). $\mathrm{N}=6$ for each group. Data is expressed as mean $\pm \mathrm{SD}$ and analyzed by $t$-test. ${ }^{*} p<0.05$ versus sham; ${ }^{*} p<0.05$ versus t-SCl $24 \mathrm{~h}$. 
Ten random neurons per section were used to count the mitochondria around the neuron nucleus in the photos at $4,200 \times$ magnification. The mitochondria vacuolization rate referred to the mean ratio of vacuolated to total mitochondria in each group.

\section{Statistical Analysis}

Data is expressed as the mean $\pm \mathrm{SD}$ and analyzed by $t$-test, one-way ANOVA, or two-way ANOVA and Bonferroni's post hoc multiple comparisons test, with $p<0.05$ suggesting statistical significance. The statistical analyses were conducted by GraphPad Prism for Windows (GraphPad, Inc., San Diego, CA, United States).

\section{RESULTS}

\section{Animals}

Major physiological data were maintained within normal ranges during the surgery and no significant differences were observed among groups (Table 2). The cardinal surgical procedures used to induce spinal cord compression injury are shown in Figures 2A-C. Representative spinal cord specimens and slices in the sham and t-SCI $24 \mathrm{~h}$ groups are shown in Figures 2D-F.

\section{Protein Levels of DJ-1 and p-Akt}

Western blotting indicated that the protein level of DJ-1 began to significantly increase at $3 \mathrm{~h}$ and peaked at $24 \mathrm{~h}$ post-injury, when it was compared with the sham group $(p<0.05)$. The protein level of p-Akt began to significantly increase at $6 \mathrm{~h}$ and peaked at $24 \mathrm{~h}$ post-injury, when it was compared with the sham group $(p<0.05)$. The protein levels of both DJ-1 and p-Akt significantly decreased after $24 \mathrm{~h}$ post-injury but were still higher than those in the sham group $(p<0.05$, Figures $3 \mathbf{A}, \mathbf{B})$.

\section{Distribution of DJ-1 in Cells}

Double IF staining indicated that DJ-1 was expressed in neurons (Figure 3C). The proportion of DJ-1-positive neurons was increased at $24 \mathrm{~h}$ post-injury, when it was compared with the sham group $(p<0.05$, Figures 3D,E).

\section{Downregulation of DJ-1 Increases Neuronal Apoptosis}

At $24 \mathrm{~h}$ post-injury, Western blotting indicated that the protein levels of DJ-1, p-Akt, and SOD2 were significantly elevated in the $\mathrm{t}$-SCI + vehicle group, compared to the sham group $(p<0.05)$. Treatment with DJ-1 siRNA significantly reduced the levels of DJ-1, p-Akt, and SOD2, compared to the t-SCI + vehicle group $(p<0.05$, Figures 4A-C). The ROS level, p-p38 MAPK/p38 MAPK ratio, and CC-3 level were significantly elevated, whereas the $\mathrm{Bcl}-2 / \mathrm{Bax}$ ratio was significantly reduced in the $\mathrm{t}$-SCI + vehicle group, compared to the sham group $(p<0.05)$. Treatment with DJ-1 siRNA significantly elevated the ROS level, p-p38 MAPK/p38 MAPK ratio, and CC-3 level and

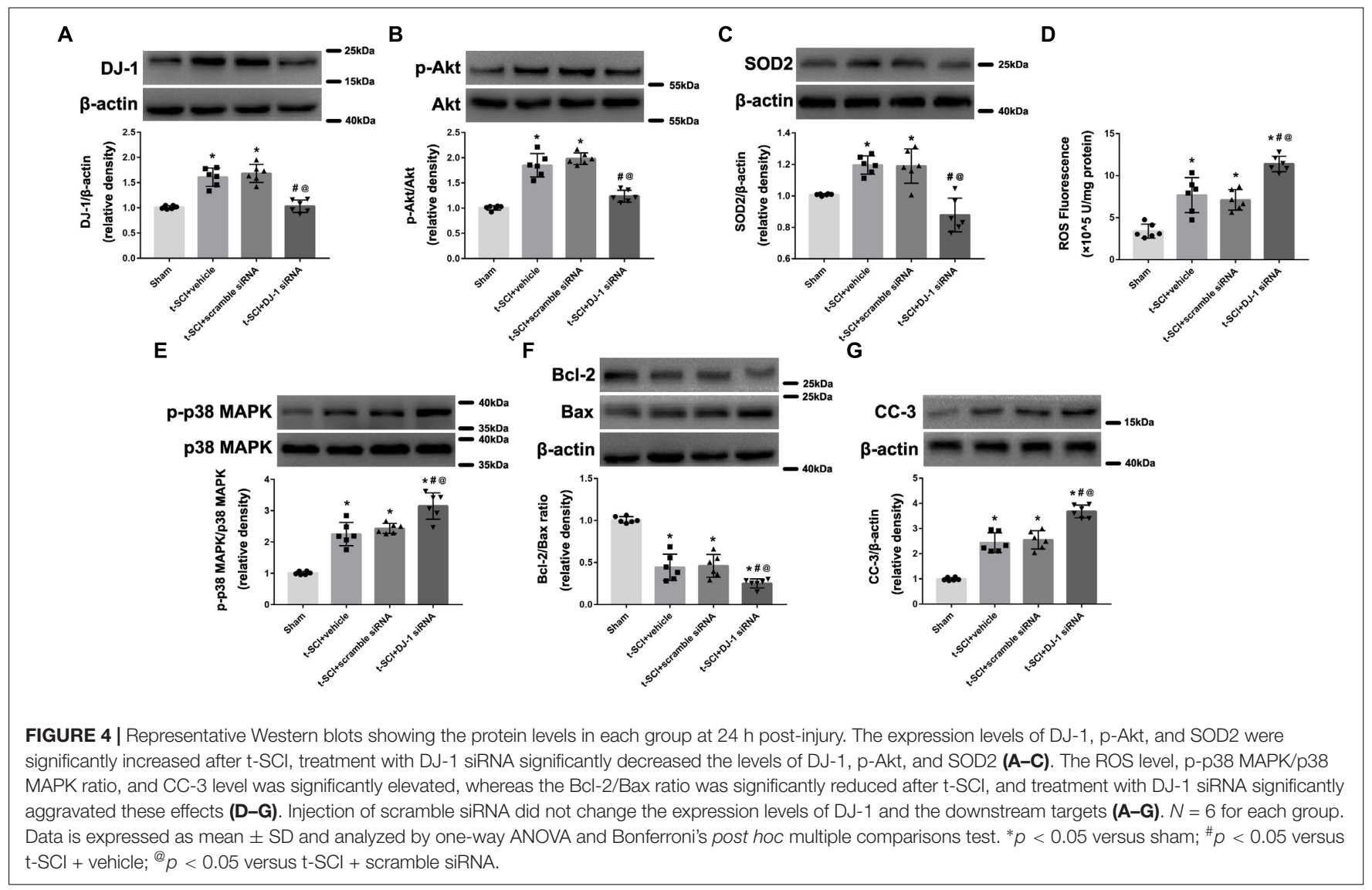


reduced the $\mathrm{Bcl}-2 / \mathrm{Bax}$ ratio, compared to the $\mathrm{t}-\mathrm{SCI}+$ vehicle group $(p<0.05$, Figures $4 \mathrm{D}-\mathbf{G})$. Treatment with scramble siRNA did not alter the protein levels of DJ-1 or its downstream targets compared to those in the t-SCI + vehicle group $(p>0.05$, Figures 4A-G).

\section{NaB Treatment Upregulates DJ-1 Expression and Reduces Neuronal Apoptosis}

At $24 \mathrm{~h}$ post-injury, Western blotting indicated that treatment with $\mathrm{NaB}$ significantly elevated the protein levels of DJ-1, p-Akt, and SOD2, compared to the t-SCI + vehicle group $(p<0.05$, Figures 5A-C). Treatment with $\mathrm{NaB}$ also significantly decreased the ROS level, p-p38 MAPK/p38 MAPK ratio, and CC-3 level but elevated the $\mathrm{Bcl}-2 / \mathrm{Bax}$ ratio, compared with the $\mathrm{t}-\mathrm{SCI}+$ vehicle groups $(p<0.05$, Figures $5 \mathrm{D}-\mathbf{G})$. These effects were obviously reversed by DJ-1 siRNA injection $(p<0.05$ t-SCI $+\mathrm{NaB}$ vs. $\mathrm{t}$-SCI $+\mathrm{NaB}+\mathrm{DJ}-1$ siRNA, Figures $5 \mathbf{A}-\mathbf{F})$. The protein levels displayed no obvious differences between the t-SCI + vehicle and t-SCI $+\mathrm{NaB}+\mathrm{DJ}-1$ siRNA groups $(p>0.05$, Figures 5A-G).

\section{OxDJ-1 Expression Is Increased After t-SCI and Reduced by NaB Treatment}

At $24 \mathrm{~h}$ post-injury, Western blotting indicated that the protein level of oxDJ-1 was significantly elevated in the t-SCI + vehicle group, compared with the sham group $(p<0.05)$. Treatment with DJ-1 siRNA significantly reduced the level of oxDJ-1, compared with the t-SCI + vehicle group $(p<0.05)$. Treatment with scramble siRNA did not alter the protein level of oxDJ1 compared to those in the t-SCI + vehicle group ( $p>0.05$, Figure 6A). Treatment with $\mathrm{NaB}$ alone or combined with DJ1 siRNA significantly decreased the protein level of oxDJ-1, compared to the t-SCI + vehicle group $(p<0.05)$. The protein level showed no obvious differences between the $\mathrm{t}-\mathrm{SCI}+\mathrm{NaB}$ and $\mathrm{t}-\mathrm{SCI}+\mathrm{NaB}+\mathrm{DJ}-1$ siRNA groups $(p>0.05$, Figure 6B).

\section{NaB Treatment Increases BBB and IPT Scores and Abates Spinal Cord Edema and BSCB Leakage}

Rats in the sham group showed a mild decreases in their BBB and IPT scores immediately post-injury, but these values rapidly returned to normal levels. The $\mathrm{BBB}$ and IPT scores of each rat in the t-SCI + vehicle group and t-SCI $+\mathrm{NaB}$ group decreased to nearly 0 just after surgery, which continuously increased during the experimental period but were still obviously lower than those of the sham group at each time point $(p<0.05)$. NaB treatment significantly elevated the $\mathrm{BBB}$ and IPT scores at 21 and 28 days after t-SCI, compared to the t-SCI + vehicle group $(p<0.05$, Figures 7A,B).

At $24 \mathrm{~h}$ post-injury, the SCWC and EB extravasation were significantly elevated in the $\mathrm{t}-\mathrm{SCI}+$ vehicle group, compared to the sham group $(p<0.05)$. NaB treatment significantly decreased

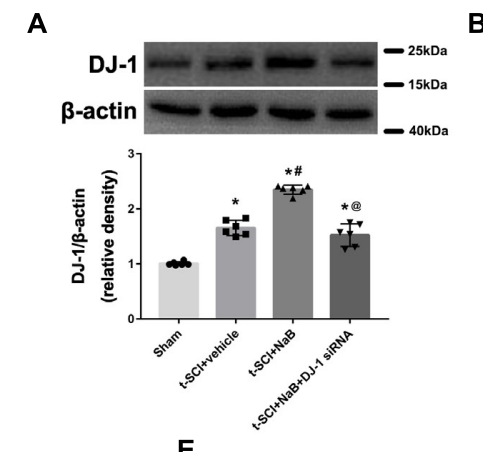

E

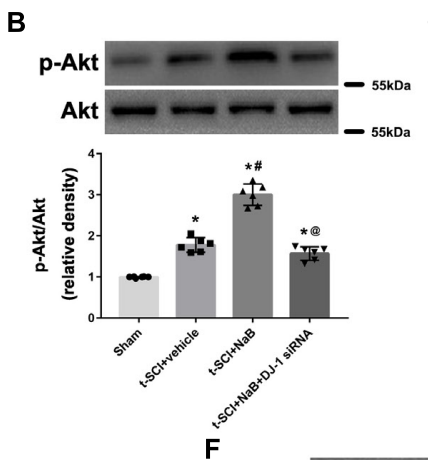

$\mathbf{F}$
C
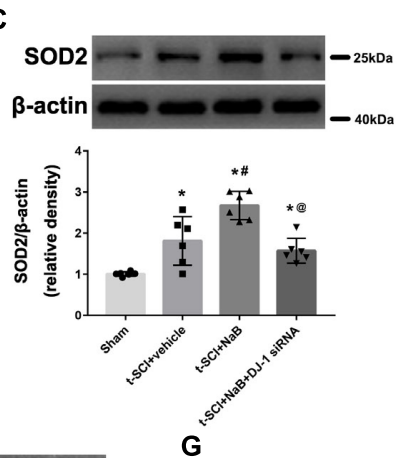

D

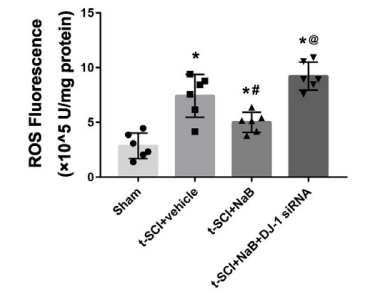

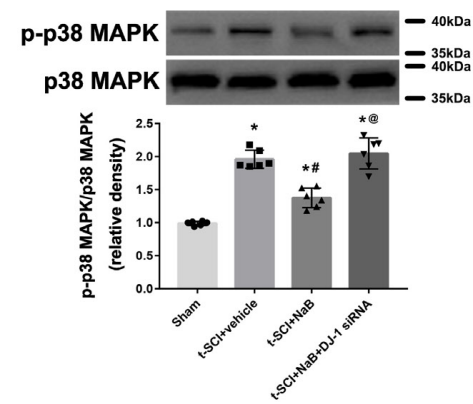
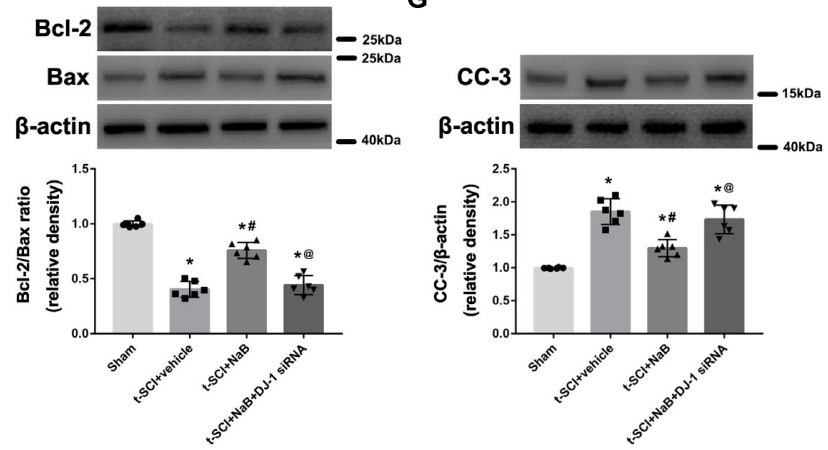

FIGURE 5 | Representative Western blots showing the protein levels in each group at $24 \mathrm{~h}$ post-injury. Treatment with NaB significantly increased the levels of DJ-1, p-Akt, and SOD2 (A-C). Treatment with NaB also significantly decreased the ROS level, p-p38 MAPK/p38 MAPK ratio, and CC-3 level and elevated the Bcl-2/Bax ratio (D-G). These effects were significantly reversed by DJ-1 siRNA injection (A-F). There were no differences in protein and ROS levels between the t-SCl + vehicle and $\mathrm{t}-\mathrm{SCl}+\mathrm{NaB}+\mathrm{DJ}-1$ siRNA groups (A-G). $N=6$ for each group. Data is expressed as mean $\pm \mathrm{SD}$ and analyzed by one-way ANOVA and Bonferroni's post hoc multiple comparisons test. ${ }^{*} p<0.05$ versus sham; ${ }^{\#} p<0.05$ versus t-SCl + vehicle; ${ }^{\circledR} p<0.05$ versus t-SCI $+\mathrm{NaB}$. 

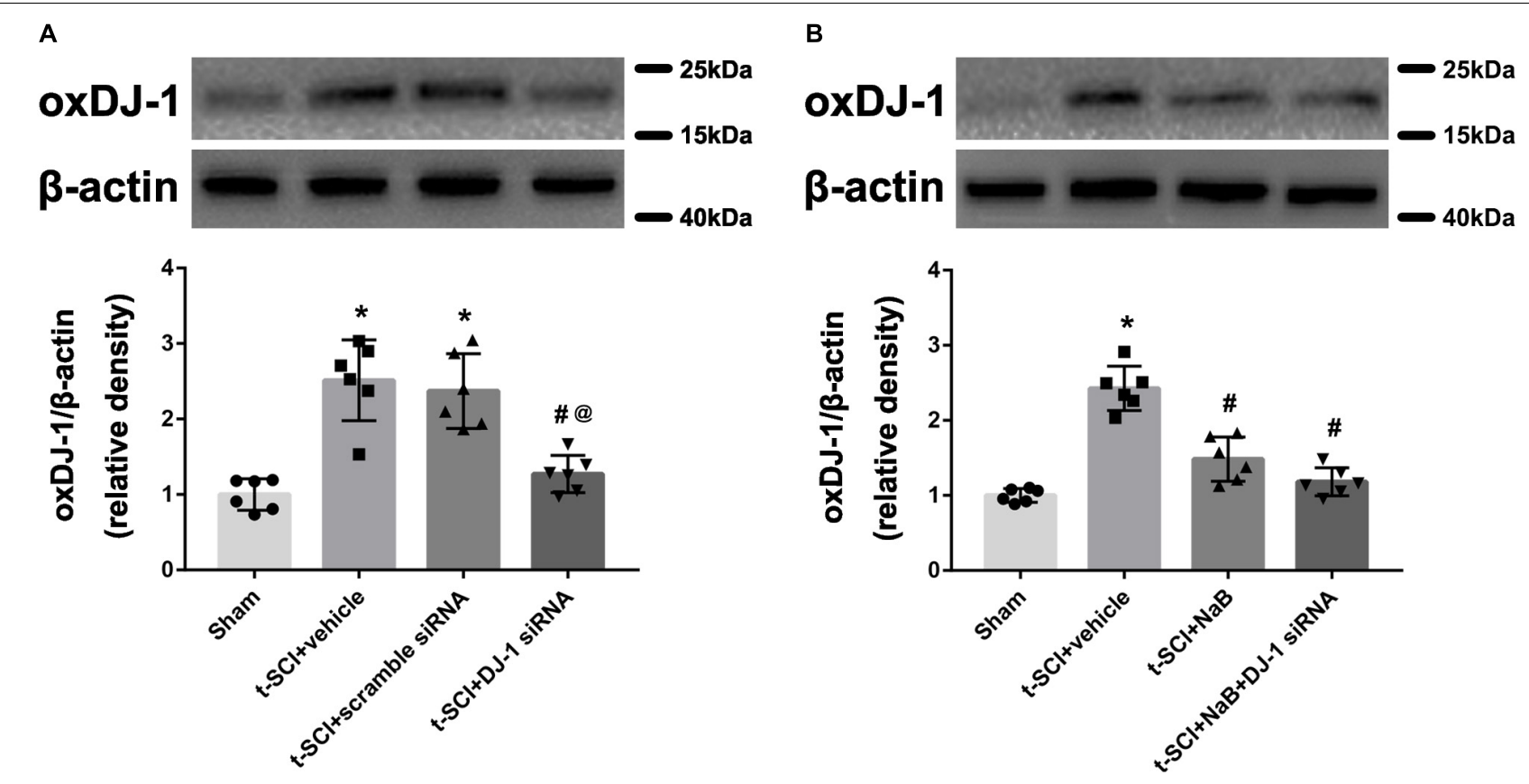

FIGURE 6 | Representative Western blots showing the protein levels in each group at $24 \mathrm{~h}$ post-injury. The expression level of oxDJ-1 was significantly increased after $\mathrm{t}-\mathrm{SCl}$, treatment with DJ-1 siRNA significantly decreased the level of oxDJ-1, and injection of scramble siRNA did not change the expression level of oxDJ-1 (A). Treatment with $\mathrm{NaB}$ alone or combined with DJ-1 siRNA significantly decreased the level of oxDJ-1. There were no differences in oxDJ-1 levels between the $\mathrm{t}-\mathrm{SCl}+\mathrm{NaB}$ and $\mathrm{t}-\mathrm{SCl}+\mathrm{NaB}+\mathrm{DJ}-1$ siRNA groups (B). $N=6$ for each group. Data is expressed as mean $\pm \mathrm{SD}$ and analyzed by one-way ANOVA and Bonferroni's post hoc multiple comparisons test. ${ }^{*} p<0.05$ versus sham; ${ }^{*} p<0.05$ versus t-SCl + vehicle; ${ }^{\circledR} p<0.05$ versus t-SCl + scramble siRNA.

the water content and EB extravasation, compared to the t-SCI + vehicle group $(p<0.05$, Figures 7C,D).

\section{Ultrastructural Changes of Spinal Cord Neurons}

At $24 \mathrm{~h}$ post-injury, the spinal cord neurons in the sham group displayed plump nuclei in which the chromatin was dispersed. Moreover, the mitochondria had a normal form and distinct crista. However, in the t-SCI + vehicle group, the nuclei of the neurons were pyknosis and the chromatin showed condensation and margination. The mitochondria also swelled and vesiculated and their crista disappeared. The aberrant ultrastructure was recovered by $\mathrm{NaB}$ treatment (Figure 8A). The proportion of mitochondrial vacuolization was significantly increased in the t-SCI + vehicle group, compared to the sham group $(p<0.05)$, which was significantly decreased by $\mathrm{NaB}$ treatment, compared to the $\mathrm{t}-\mathrm{SCI}+$ vehicle group $(p<0.05$, Figure $8 \mathrm{~B})$.

\section{NaB Reduces Neuronal Apoptosis Through DJ-1/Akt-Related Anti-oxidative Stress Cascade}

At $24 \mathrm{~h}$ post-injury, Western blotting indicated that $\mathrm{NaB}$ treatment significantly elevated the protein level of DJ-1, compared to the t-SCI + vehicle group $(p<0.05)$, and this upregulation was not altered by MK2206 treatment (Figure 9A). Treatment with $\mathrm{NaB}$ also significantly elevated the protein levels of p-Akt and SOD2, which reduced the level of ROS, compared to the t-SCI + vehicle group $(p<0.05)$; however, these effects were obviously reversed by MK2206 treatment $(p<0.05$ t-SCI $+\mathrm{NaB}+\mathrm{MK} 2206$ vs. $\mathrm{t}-\mathrm{SCI}+\mathrm{NaB}$, Figures 9BD). The p-p38 MAPK/p38 MAPK ratio and protein level of CC-3 were decreased, while the $\mathrm{Bcl}-2 / \mathrm{Bax}$ ratio was elevated under $\mathrm{NaB}$ treatment, compared to the $\mathrm{t}-\mathrm{SCI}+$ vehicle group $(p<0.05)$; however, these effects were obviously reversed by MK2206 treatment $(p<0.05$ t-SCI $+\mathrm{NaB}+\mathrm{MK} 2206$ vs. t-SCI + $\mathrm{NaB}$, Figures 9E-G). Moreover, administration of MK2206 alone did not obviously alter protein and ROS levels compared to those in the t-SCI + vehicle group ( $p>0.05$, Figures 9A-G).

At $24 \mathrm{~h}$ post-injury, double IF staining indicated that $\mathrm{t}$-SCI caused an increase in the proportions of CC-3- and TUNELpositive neurons, compared to the sham group $(p<0.05)$. However, they were decreased following $\mathrm{NaB}$ treatment compared to those in the t-SCI + vehicle group $(p<0.05)$. These effects were obviously reversed by MK2206 treatment $(p<0.05$ t-SCI $+\mathrm{NaB}+\mathrm{MK} 2206$ vs. t-SCI $+\mathrm{NaB})$. Moreover, MK2206 alone did not obviously alter the proportions of CC-3and TUNEL-positive neurons compared to those in the t-SCI + vehicle group ( $p>0.05$, Figures 10A,B, 11A,B).

\section{DISCUSSION}

As a multi-functional protein, DJ-1 is involved in diverse signaling pathways, the most important being helping cells to resist oxidative stress and oxidative stress-induced 
A

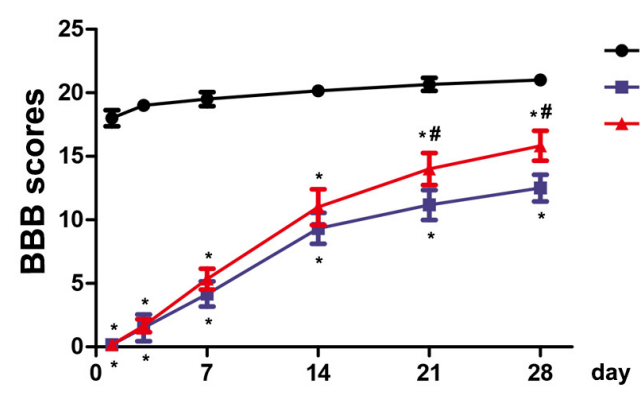

C

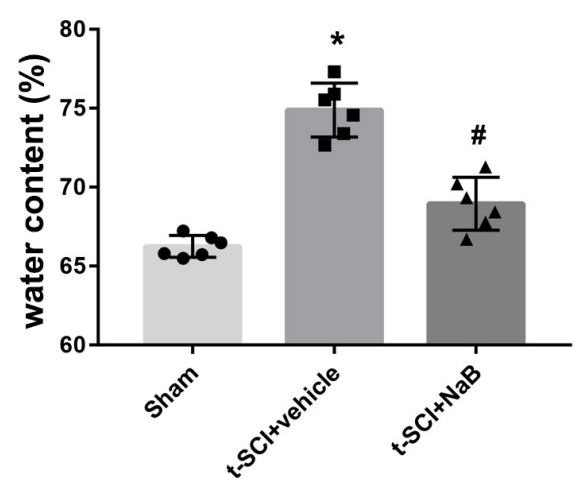

B

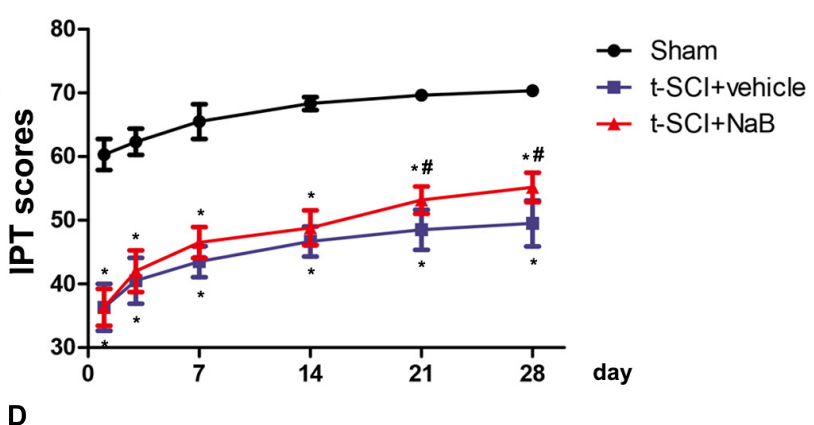

D

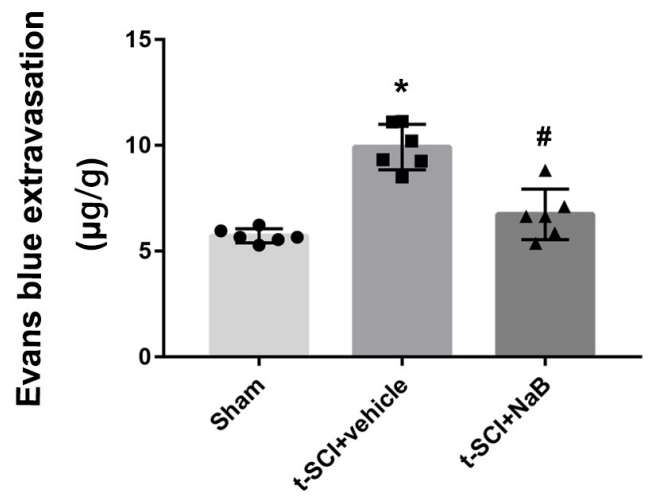

FIGURE 7 | BBB and IPT scores were significantly decreased after t-SCl at each time point. Treatment with NaB significantly increased the BBB and IPT scores at days 21 and 28 post-injury (A,B). $N=6$ for each group. Data is expressed as mean \pm SD and analyzed by two-way ANOVA and Bonferroni's post hoc multiple comparisons test. At $24 \mathrm{~h}$ post-injury, treatment with $\mathrm{NaB}$ significantly reduced the SCWC (C) and EB extravasation (D), which was increased after t-SCl. $N=6$ for each group. Data is expressed as mean $\pm \mathrm{SD}$ and analyzed by one-way ANOVA and Bonferroni's post hoc multiple comparisons test. ${ }^{*} p<0.05$ versus sham; $\# p<0.05$ versus $\mathrm{t}-\mathrm{SCl}+$ vehicle.

apoptosis (Pantcheva et al., 2014), which is responsible for its neuroprotective functions in multiple neurological diseases. In an animal model of ischemia-reperfusion SCI, the expression of DJ-1 was increased significantly at the early stage of reperfusion (Sakurai et al., 2009). Injection of DJ-1 fusion protein protected ischemic spinal cord injury neurons by increasing the levels of antioxidant protein in cells, but the specific mechanism is unclear (Kim et al., 2014). In contrast, DJ-1 knockout makes cells vulnerable to oxidative stress (Taira et al., 2004). However, its protective effects on t-SCI and its precise molecular mechanisms remain unclear. Oxidative stress following t-SCI is a major factor causing damage to neurons. Diverse traumatic and non-traumatic SCI causes the generation of excessive ROS and dysregulation of redox homeostasis, which contribute to pathological injury to the spinal cord (Zhang et al., 2015).

Oxidative stress-induced neuronal damage is mediated by diverse mechanisms, one of which is the apoptotic cascade. ROS can lead to cell apoptosis by activating the p38 MAPK signaling pathway (Bode et al., 2012). Gomez-Lazaro et al. (2007) suggested that ROS production activated p38 MAPK and subsequently promoted the activation of the pro-apoptotic protein Bax, which can penetrate the mitochondrial membrane to cause the release of cytochrome $\mathrm{C}$ and activate the intrinsic apoptosis pathway in SH-SY5Y cells. Choi et al. (2004) reported that ROS initiated the p38 MAPK signaling pathway, leading to the activation of both mitochondrial and extra-mitochondrial apoptotic pathways in PD cell models. Ghatan et al. (2000) reported that p38 MAPK played a key role in NO-mediated neuronal death by stimulating the translocation of Bax to the mitochondria, which in turn triggered the apoptotic cascade. Neuronal apoptosis is one of the factors contributing to the poor prognosis of t-SCI (Niu and Yip, 2011). After t-SCI, some cells at the lesion site die because of post-traumatic necrosis, whereas others die by apoptosis (Byrnes et al., 2007). The apoptotic cascade can be activated in oligodendrocytes, microglia, and perhaps, astrocytes, and particularly in neurons (Liu et al., 1997; Beattie et al., 2000), which may be responsible for the serious dysfunction of patients with t-SCI (Mizuno et al., 1998; Mattson, 2000). Previous studies revealed that decreased apoptosis can promote functional improvement in t-SCI rats (Wang et al., 2017). DJ1 exerts anti-apoptotic effects by reducing ROS. Additionally, DJ-1 can modulate the p38 MAPK pathway via direct physical interactions with its upstream protein, the ASK1, to reduce apoptosis (Mo et al., 2010).

In the present study, Western blotting indicated that DJ-1 was significantly increased after t-SCI induction. The ROS level, p-p38 MAPK/p38 MAPK ratio, and CC-3 level was significantly elevated, whereas the $\mathrm{Bcl}-2 / \mathrm{Bax}$ ratio was significantly reduced after t-SCI. Furthermore, treatment with DJ-1 siRNA significantly downregulated the protein level of 

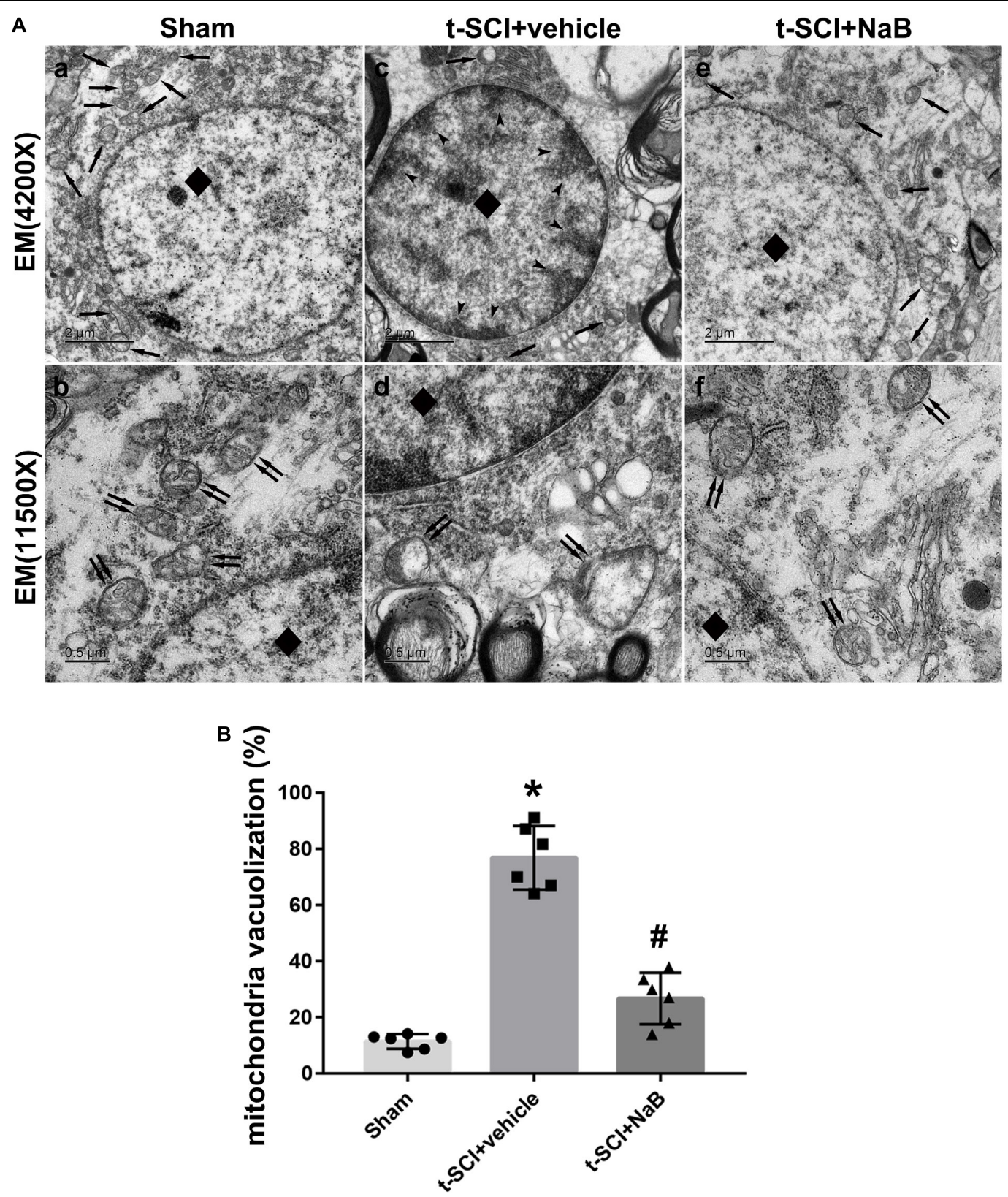

FIGURE 8 | Representative TEM micrographs showing ultrastructural changes of spinal cord neurons in each group at $24 \mathrm{~h}$ post-injury. Normal nucleus (diamond) and mitochondrion (arrows) in the sham group (A, a); normal mitochondrion (double arrows) with clear crista in the sham group (A, b); karyopyknosis (diamond), chromatin condensation, and margination (dovetail arrows), disappearance of the nucleolus, mitochondrial loss (arrows) in the $\mathrm{SCl}+\mathrm{vehicle} \mathrm{group} \mathrm{(A,} \mathrm{c);}$ mitochondrial swelling, vesiculation, and disappearance of the crista (double arrows) in the $\mathrm{SCl}+$ vehicle group (A, d); treatment with $\mathrm{NaB}$ reversed neuronal apoptosis, and the subcellular structure reverted to near normal (A, e,f). $N=6$ for each group. (B) The proportion of mitochondrial vacuolization significantly increased after $\mathrm{t}-\mathrm{SCl}, \mathrm{NaB}$ treatment significantly decreased the proportion of mitochondrial vacuolization. Data is expressed as mean $\pm \mathrm{SD}$ and analyzed by one-way ANOVA and Bonferroni's post hoc multiple comparisons test. ${ }^{*} p<0.05$ versus sham; ${ }^{\#} p<0.05$ versus t-SCl + vehicle.

DJ-1, elevated the ROS level, p-p38 MAPK/p38 MAPK ratio, and CC-3 level, and reduced the Bcl-2/Bax ratio. These results indicated that initiation of the ROS-induced apoptosis occurred after t-SCI, and DJ-1 showed preventative effects on ROSinduced apoptosis and functioned as a neuroprotective protein. Treatment with $\mathrm{NaB}$ significantly increased the expression level of DJ-1. Moreover, $\mathrm{NaB}$ treatment significantly decreased the
ROS level, p-p38 MAPK/p38 MAPK ratio, and CC-3 level and elevated the Bcl-2/Bax ratio. DJ-1 siRNA significantly reversed these beneficial effects. TEM analysis also revealed abnormal subcellular structures and an increased proportion of mitochondrial vacuolization in t-SCI + vehicle group, indicating that neuronal apoptosis was induced by t-SCI. NaB treatment reversed t-SCI-induced apoptosis to some extent. The results 


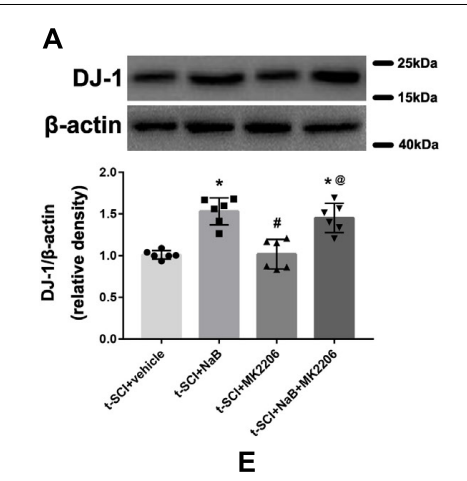

$E$
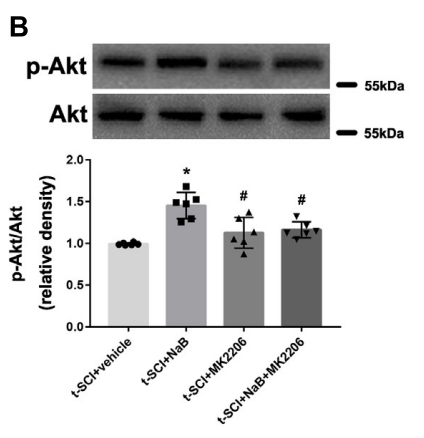

$F$
C
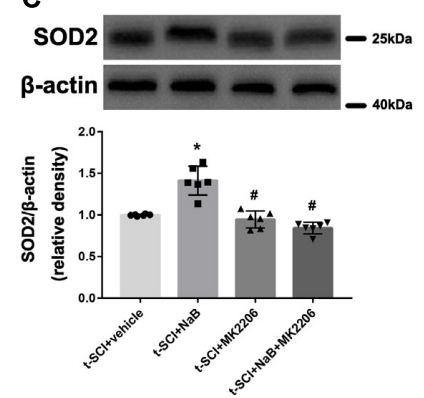

G
D

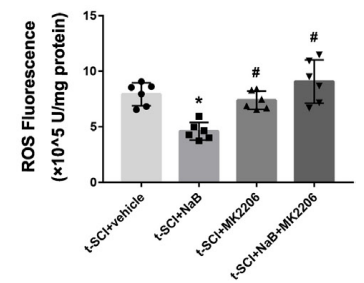

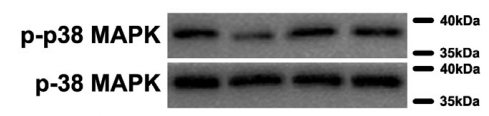
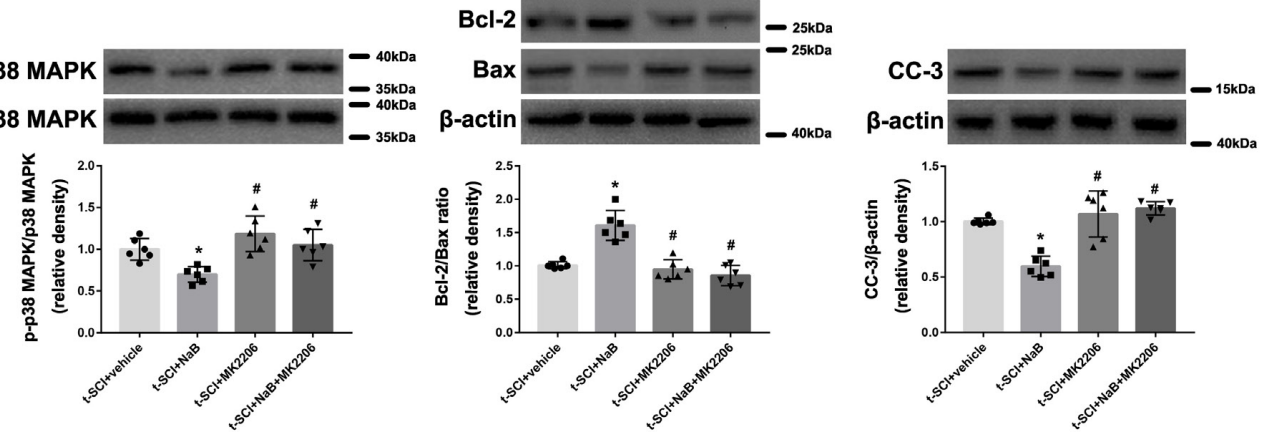

FIGURE 9 | Representative Western blots showing the protein levels in each group at 24 h post-injury. Treatment with NaB significantly increased the level of DJ-1, and this upregulation was not affected by treatment with MK2206 (A). The increase in the levels of p-Akt and SOD2, induced by NaB, were reversed by MK2206 (B,C). The decrease in the ROS level, p-p38 MAPK/p38 MAPK ratio, and CC-3 level and the increase in the Bcl-2/Bax ratio, induced by NaB, were reversed by MK2206 (D-G). Administration of MK2206 alone did not significantly alter the levels of DJ-1, its downstream proteins, and ROS (A-G). $N=6$ for each group. Data is expressed as mean \pm SD and analyzed by one-way ANOVA and Bonferroni's post hoc multiple comparisons test. * $p<0.05$ versus t-SCI + vehicle; ${ }^{*} p<0.05$ versus t $-\mathrm{SCl}+\mathrm{NaB} ;{ }^{\circledR} p<0.05$ versus t-SCI + $\mathrm{MK} 2206$.

confirmed that $\mathrm{NaB}$ treatment alleviated ROS-induced apoptosis by upregulating DJ-1 expression, which agrees with the results of previous studies and further supports the crucial anti-apoptotic effects of DJ-1 in t-SCI rats.

The ability to respond to oxidative stress is the best-established characteristic of DJ-1 (Canet-Aviles et al., 2004). Under oxidative stress, DJ-1 is transformed to a cysteine sulfinic acid (Cys106$\mathrm{SO}_{2}$ ) via oxidation of its Cys106 residue as a post-translational modification (Blackinton et al., 2009b). It was reported that oxidation of Cys106 of DJ-1 contributed to its protective effects, while the absence of Cys106 oxidation led to the loss of DJ1's protective function (Blackinton et al., 2009b). Additionally, excessive oxDJ-1 enables cells to commit to apoptosis (Cao et al., 2014). In patients and animal models of $\mathrm{PD}, \mathrm{oxDJ}-1$ was increased in unmedicated PD, while drug therapy lowered oxDJ-1 levels, suggesting oxDJ-1 played an important role in $\mathrm{PD}$ and was a potential biomarker for PD (Saito, 2014; Saito et al., 2014; Mita et al., 2018; Yamagishi et al., 2018). Thus, we also tested the expression of oxDJ-1 and found that oxDJ-1 was significantly increased after t-SCI induction. DJ-1 siRNA, NaB, or $\mathrm{NaB}+\mathrm{DJ}$ 1 siRNA significantly reduced the expression of oxDJ-1. Under t-SCI, DJ-1 can be oxidized into oxDJ-1, resulting in elevation of oxDJ-1 levels. DJ-1 siRNA reduced the levels of DJ-1 and oxDJ-1. DJ-1 upregulated by $\mathrm{NaB}$ can reduce ROS through self-oxidation (Taira et al., 2004) and form oxDJ-1, which was thought to cause upregulation oxDJ-1. However, we found a decrease in oxDJ-1 after $\mathrm{NaB}$ treatment. These results indicated that $\mathrm{NaB}$ treatment elevated the level of DJ-1 and eliminated ROS through other more powerful pathways, which reduced the oxidation of ROS toward DJ-1 and led to a decrease in oxDJ-1. In fact, self-oxidation is not the main antioxidant mechanism and contributes little to the antioxidant capacity of DJ-1 (Aleyasin et al., 2010).

Apart from neuronal apoptosis, t-SCI also induces edema and BSCB breakdown, which are the major pathological changes and contribute to a poor prognosis (Gao et al., 2018). In the clip compressive model of t-SCI, the compression forces applied to spinal cord can mutilate blood vessels, damage cellular components, and breakdown BSCB (Casella et al., 2006; Dulmovits and Herman, 2012). Sharma (2011) suggested that early spinal cord microvascular reactions after t-SCI led to spinal cord endothelial cell disturbances and caused the breakdown of BSCB functions. Destruction of the BSCB alters the microenvironment of the spinal cord (Wu et al., 2014) and thus causes the soakage of massive neutrophils and macrophages, contributing to severe inflammation, cell death, and durable neurological dysfunctions (Lee et al., 2012). In contrast, a reduction in $\mathrm{BSCB}$ breakdown results in significant neuroprotection (Wu et al., 2014). Spinal cord edema results from excessive SCWC both in the extracellular and intracellular spaces which can result from trauma, ischemia, and inflammation (Nesic et al., 2006). There are two main types of spinal cord edema: cytotoxic edema and vasogenic edema. T-SCI causes 


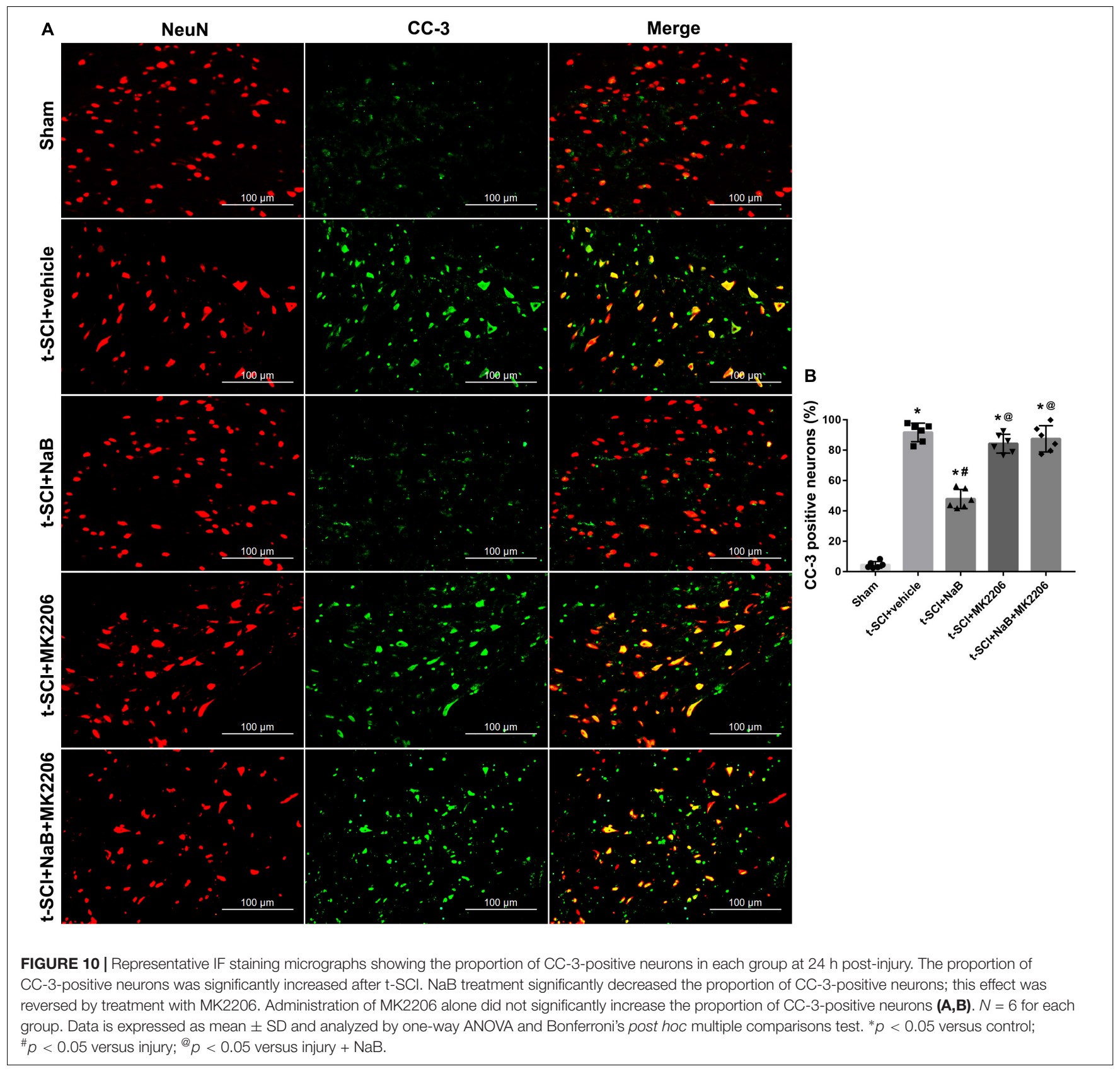

perivascular astrocyte endfeet swelling and dysfunction. As a result, excessive water enters the cells and leads to cytotoxic edema, further destroying the tight junction and vascular basal lamina, causing water to exudate from blood vessels and form vasogenic edema (Wang et al., 2011). Additionally, spinal cord edema can exacerbate destruction of the BSCB. In other words, spinal cord edema and BSCB destruction may exhibit reciprocal causation (Nesic et al., 2006). In our study, we found that t-SCI led to an increase in SCWC and EB extravasation, indicating edema and BSCB destruction of the injured spinal cord. However, these effects were significantly reversed by $\mathrm{NaB}$ treatment.

Overall, $\mathrm{NaB}$ treatment elevated the expression level of DJ-1, and then effectively reduced ROS-induced neuronal apoptosis. Additionally, $\mathrm{NaB}$ reduced spinal cord edema and $\mathrm{BSCB}$ destruction. At the macro level, the decreases in the $\mathrm{BBB}$ and IPT scores induced by t-SCI were significantly increased by $\mathrm{NaB}$ treatment at 21- and 28-days post-injury, indicating improvement in neurological functions. However, the macroscopic functional improvement results from a combination of multiple micro-events, which are not limited to the alleviation of neuronal apoptosis, spinal cord edema, or BSCB destruction. As a result, the improvement in neurological function is delayed in time with respect to improvement at the molecular and cellular levels as shown in our experiment. This indicates that other pathogenic factors contribute to the neurological impairment of $\mathrm{t}-\mathrm{SCI}$, which requires further analysis. 


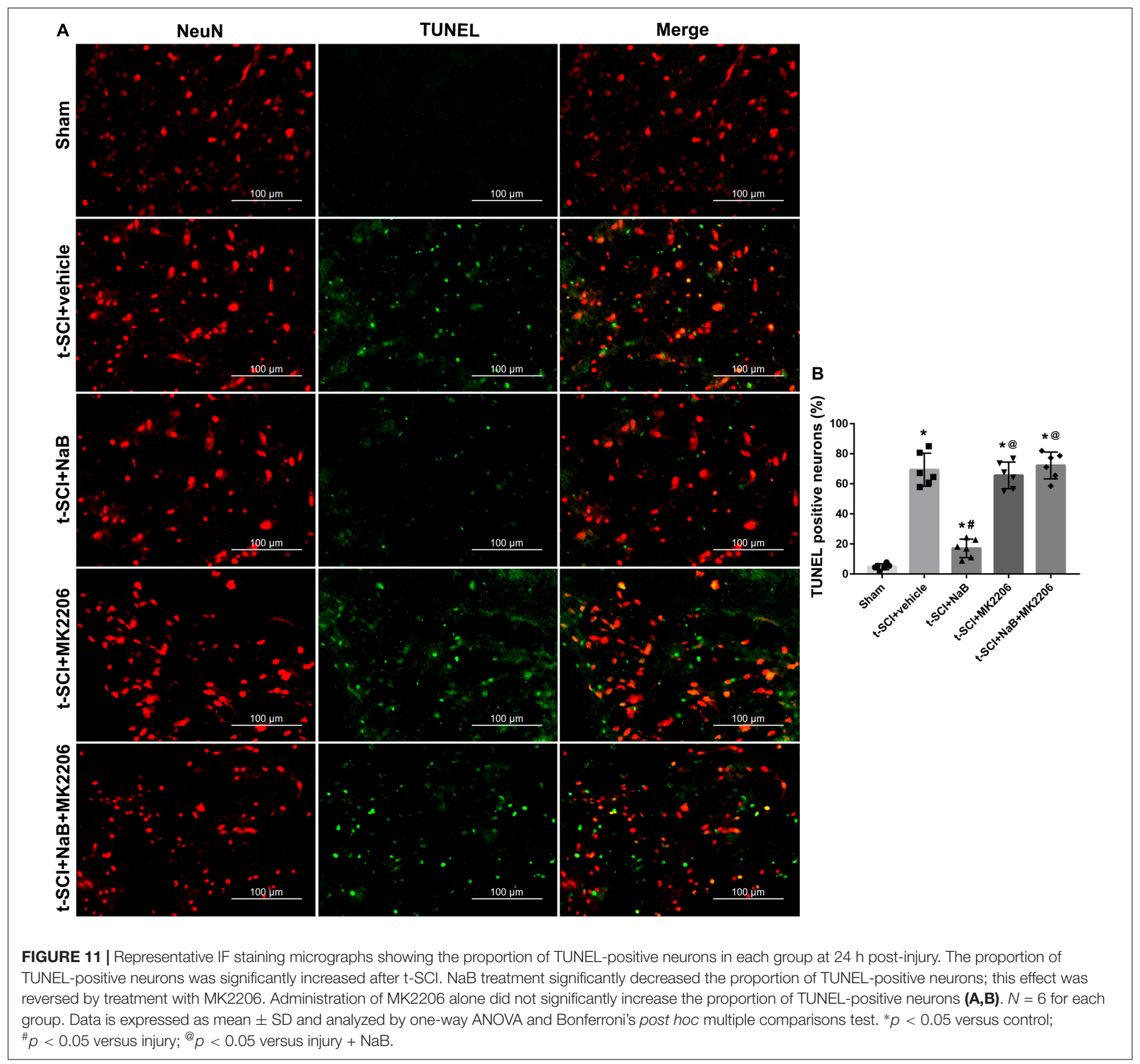

The DJ-1 gene belongs to the Thi/PfpI superfamily (Wang et al., 2018), which is located at chromosome 1p36 and encodes a ubiquitous protein consisting of 189 amino acids (Van Duijn et al., 2001). Among its biological functions, the most important is protecting against oxidative stress (Kahle et al., 2009). The main anti-oxidative stress mechanisms of DJ-1 include multiple aspects. First, DJ-1 is a redox protein; it removes ROS in vitro and in vivo by self-oxidation (Taira et al., 2004). The expression of DJ-1 is induced by oxidative stresses (Kinumi et al., 2004). In the three cysteine residues of DJ-1, Cys106 is the most sensitive amino acid toward intracellular oxidative stress. DJ-1 scavenges ROS when the Cys106 residue is oxidized to the acid subtype (Canet-Aviles et al., 2004). Second, DJ-1 shows molecular chaperone activity that is sensitive to redox reaction, helping cells resist harmful events induced by oxidative stress (Zhou et al., 2006). Third, DJ-1 acts as a transcriptional coactivator that can promote the transcription of glutathione and SOD (Zhong and $\mathrm{Xu}, 2008$ ) and regulate the activity of peroxiredoxin-2, a significant antioxidant enzyme (Qu et al., 2007), which in turn decreases ROS levels. It has also been reported that nuclear factor erythroid 2-related factor-2, a transcription factor, can be stabilized by DJ-1, promoting its shift from the cytoplasm to the nucleus and then upregulating the expression of antioxidant genes (Malhotra et al., 2008). Fourth, DJ-1 interacts with several regulatory molecules in the nucleus to exert synergistic transcriptional regulatory actions (Sekito et al., 2006). Fifth, DJ-1 can modulate the function of mitochondria by maintaining the activity of mitochondrial complex 1, reducing ROS in 
the mitochondria, and preserving the mitochondrial membrane potential and mitochondrial shape (Krebiehl et al., 2010). The activity of mitochondrial complex 1 was downregulated in DJ-1 knockdown cells (Canet-Aviles et al., 2004; Ooe et al., 2005) and DJ-1 knockout mice (Goldberg et al., 2005; Kim et al., 2005b).

Moreover, previous studies showed that DJ-1 promoted the phosphorylation of Akt for activation, which in turn protected against oxidative stress damage. Akt is a serine/threonine protein kinase that participates in many aspects of cell activity including cell survival, growth, proliferation, differentiation, metabolism, and death (Manning and Toker, 2017). Akt is involved in diverse physical and pathological processes during CNS injuries (Ko et al., 2016). Additionally, Akt has many phosphorylation sites, among which the phosphorylation of Ser473 in the hydrophobic motif causes maximal activation (Manning and Toker, 2017). It was also reported that DJ-1 promoted the phosphorylation of Akt at Ser473 (Kim et al., 2005a; Aleyasin et al., 2010; Wang et al., 2014). As a result, we examined Ser473 phosphorylation of Akt in this study. Western blotting indicated that the expression level of DJ-1 was increased starting at $3 \mathrm{~h}$ after t-SCI, reached a peak at $24 \mathrm{~h}$, and then gradually decreased at 48 and $72 \mathrm{~h}$. Interestingly, the p-Akt levels showed a similar trend. Additionally, the p-Akt level was decreased following knockdown of DJ-1, while it was increased following an increase in DJ-1 after $\mathrm{NaB}$ treatment. However, some studies showed that DJ1 promoted the phosphorylation of Akt at other sites, such as Ser505 (Yang et al., 2005) and Thr308 (Gorner et al., 2007; Zhang et al., 2016), which were also involved in preventing oxidative stress. Whether phosphorylation at these sites is important remains unclear and requires further analysis.

Activated Akt can help multiple types of cells to resist ROS-induced injuries via diverse pathways, as well as by modulating the expression of antioxidant enzymes. The major antioxidant enzymes include glutathione peroxidase, catalase, and particularly the SOD family, which help to degrade ROS to reduce damage to DNA, proteins, lipids, and other cellular components (Davis and Pennypacker, 2017). The SOD family has three isoforms, SOD1, SOD2, and SOD3, among which SOD2 is ubiquitously expressed and one of the major antioxidant enzymes responsible for scavenging ROS in the mitochondria (Chan, 2005). Previous studies reported that decreased SOD2 activity was associated with $\mathrm{AD}$ and PD (Wiener et al., 2007; Belluzzi et al., 2012). SOD2 knockout mice were more sensitive to oxidative stress after cerebral ischemia (Kim et al., 2002; Mehta et al., 2011), while increased SOD2 alleviated ischemic brain injury (Davis and Pennypacker, 2017). Akt activation promoted the expression of SOD2 to exert cell protective effects (Saha et al., 2016). As a result, we detected SOD2 in our study and found that SOD2 expression was consistent with p-Akt expression.

These results suggested that upregulation of DJ-1 promoted the expression of SOD2 by activating Akt, which in turn disintegrated ROS in rats with t-SCI. As a result, ROSinduced apoptosis was also reduced. We further utilized the highly selective Akt inhibitor MK2206 to confirm these results. MK2206 effectively inhibits the phosphorylation of Akt, and thus prevents the activation of downstream molecules. Western blotting showed that MK2206 treatment significantly blocked the anti-oxidative stress effects of DJ-1. Double IF staining showed that the proportions of CC-3- and TUNEL-positive neurons were increased significantly post-injury, indicating neuronal apoptosis. Treatment with $\mathrm{NaB}$ significantly reduced neuronal apoptosis; however, this decrease was reversed by MK2206. Notably, given that MK2206 alone did not obviously alter neuronal apoptosis, confirming mediation of the protective effects of DJ-1 via phosphorylation of Akt. The reason for this may be that MK2206 could not inhibit Akt under injury without additional interventions.

The results of our study suggested that $\mathrm{NaB}$ reduced ROS and ROS-induced neuronal apoptosis by upregulating DJ-1 and its downstream proteins, p-Akt and SOD2. A schematic map of the potential signaling pathway is shown in Figure 12.

$\mathrm{NaB}$ is a metabolite of cinnamon and FDA-approved food additive, which has wide applications in the food industry. It is non-toxic and can be administered as a solution in drinking water. Long-term intake of a $2 \% \mathrm{NaB}$ aqueous solution is safe and shows no side effects in experimental mice (Toth, 1984). A minor amount of $\mathrm{NaB}$ is expelled in the urine of humans after intake (Kubota and Ishizaki, 1991). The typical FDA-approved

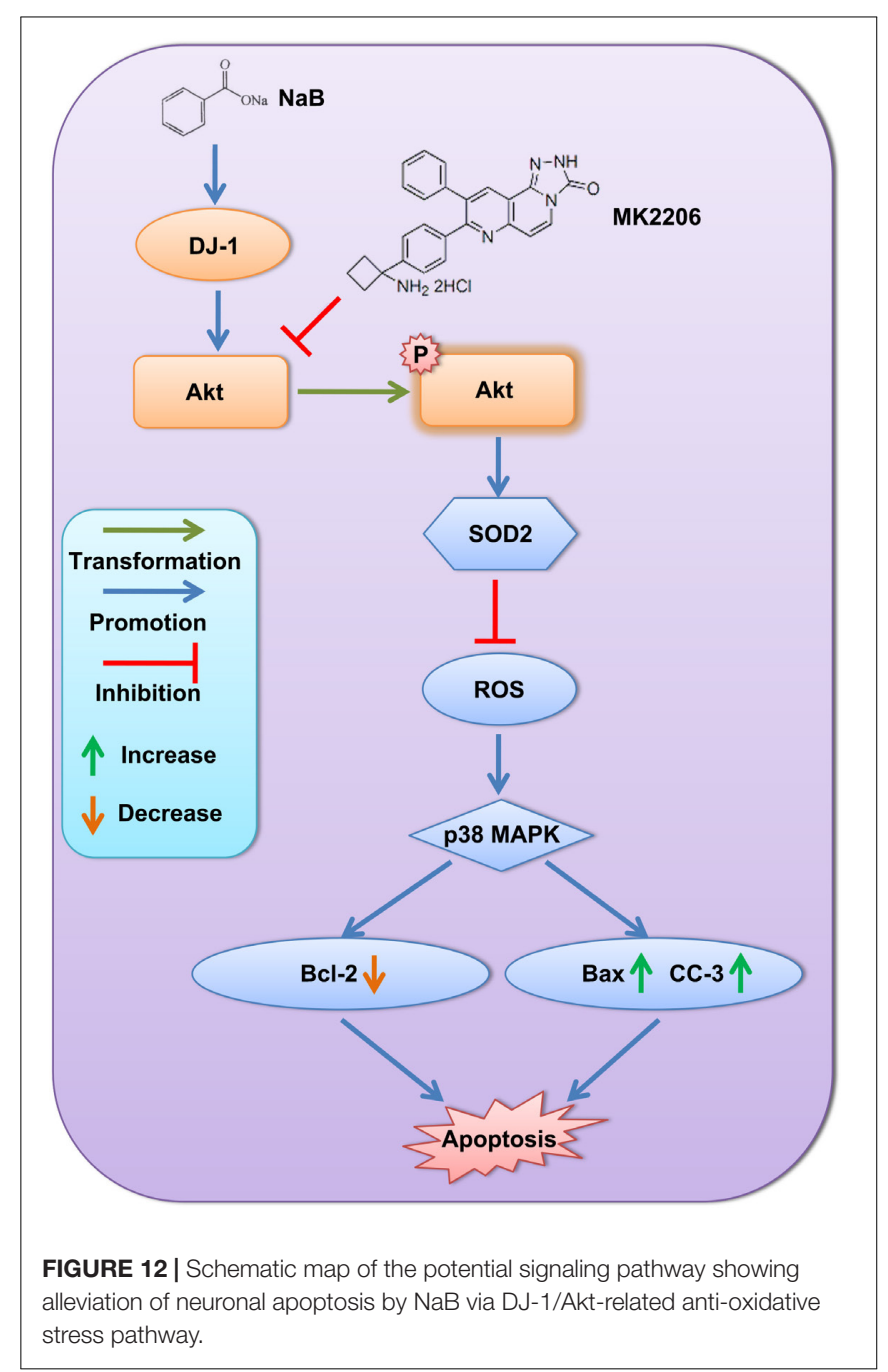


dose of $\mathrm{NaB}$ for patients with hyperammonemia is $4-10 \mathrm{~g} / \mathrm{day}$, which was further increased during treatment of acute stage hyperammonemia (Sushma et al., 1992; Misel et al., 2013). As a potential drug for treating $\mathrm{CNS}$ disorders, $\mathrm{NaB}$ shows obvious advantages. First, $\mathrm{NaB}$ can be administered orally, which is convenient and results in good compliance; second, $\mathrm{NaB}$ is a liposoluble molecule capable of diffusing through the BSCB. $\mathrm{NaB}$ shows considerable accumulation in the cerebrospinal fluid, making it an effective agent for treating CNS disorders (Khasnavis and Pahan, 2012). However, the optimal NaB dosage and duration of treatment of CNS injury, particularly for t-SCI requires further analysis.

\section{LIMITATIONS}

There were some limitations to our study. First, DJ-1 can exert neuroprotective effects through a variety of pathways; such pathways are often linked with each other, whereas we only studied the anti-oxidative stress and anti-ROS-induced apoptosis effects. Further studies are needed to evaluate other mechanisms. Second, in our study, $\mathrm{NaB}$ treatment alleviated spinal cord edema and BSCB destruction; however, its exact molecular mechanism and the mechanism of action of DJ-1 should be further examined. Third, in addition to neurons, large numbers of white matter fibers exist in the spinal cord. As a result, white matter injury is also a critical pathological process during t-SCI. Whether DJ-1 is involved in preventing white matter injury should be further evaluated.

\section{CONCLUSION}

This is the first study to demonstrate the neuroprotective role of $\mathrm{NaB}$ in a rat model of $\mathrm{t}-\mathrm{SCI}$. $\mathrm{NaB}$ upregulated $\mathrm{DJ}-1$, and

\section{REFERENCES}

Advedissian, T., Deshayes, F., Poirier, F., Viguier, M., and Richarme, G. (2016). The Parkinsonism-associated protein DJ-1/Park7 prevents glycation damage in human keratinocyte. Biochem. Biophys. Res. Commun. 473, 87-91. doi: 10.1016/ j.bbrc.2016.03.056

Ahuja, C. S., Wilson, J. R., Nori, S., Kotter, M. R. N., Druschel, C., Curt, A., et al. (2017). Traumatic spinal cord injury. Nat. Rev. Dis. Primers 3:17018. doi: $10.1038 /$ nrdp. 2017.18

Aleyasin, H., Rousseaux, M. W., Marcogliese, P. C., Hewitt, S. J., Irrcher, I., Joselin, A. P., et al. (2010). DJ-1 protects the nigrostriatal axis from the neurotoxin MPTP by modulation of the AKT pathway. Proc. Natl. Acad. Sci. U.S.A. 107, 3186-3191. doi: 10.1073/pnas.0914876107

Aleyasin, H., Rousseaux, M. W., Phillips, M., Kim, R. H., Bland, R. J., Callaghan, S., et al. (2007). The Parkinson's disease gene DJ-1 is also a key regulator of strokeinduced damage. Proc. Natl. Acad. Sci. U.S.A. 104, 18748-18753. doi: 10.1073/ pnas.0709379104

Bandopadhyay, R., Kingsbury, A. E., Cookson, M. R., Reid, A. R., Evans, I. M., Hope, A. D., et al. (2004). The expression of DJ-1 (PARK7) in normal human CNS and idiopathic Parkinson's disease. Brain 127, 420-430. doi: 10.1093/brain/ awh054

Basso, D. M., Beattie, M. S., and Bresnahan, J. C. (1995). A sensitive and reliable locomotor rating scale for open field testing in rats. J. Neurotrauma 12, 1-21. doi: $10.1089 /$ neu.1995.12.1 thus reduced ROS and ROS-induced neuronal apoptosis via Akt phosphorylation, and increased SOD2 expression. Given the crucial involvement of ROS in t-SCI, DJ-1 may be an important target while $\mathrm{NaB}$ is a promising therapeutic agent for treating $\mathrm{t}-\mathrm{SCI}$.

\section{ETHICS STATEMENT}

This study was carried out in accordance with the recommendations of the NIH guidelines. The protocol was approved by the ethics committee of Zhejiang University.

\section{AUTHOR CONTRIBUTIONS}

BQ, JZ, TL, and GY performed the analysis of the t-SCI model. FY, WX, ZZ, and BQ performed the Western blots. TZ and JZ prepared the figures. LG and GY performed the IF staining. TL and JL performed the data analysis. LG, YZ, and GC designed the experiments. LG, ZZ, and WX contributed to the writing and editing of the manuscript. YZ and GC reviewed and revised the manuscript.

\section{FUNDING}

This work was supported by the National Natural Science Foundation of China (Grant No. 81771246). The project was cosponsored by the Natural Science Foundation of Zhejiang Province (Grant No. LY18H090005) and Key Projects of Traditional Chinese Medicine of Zhejiang Province (Grant No. 2017ZZ013).

Batelli, S., Albani, D., Rametta, R., Polito, L., Prato, F., Pesaresi, M., et al. (2008). DJ1 modulates alpha-synuclein aggregation state in a cellular model of oxidative stress: relevance for Parkinson's disease and involvement of HSP70. PLoS One 3:e1884. doi: 10.1371/journal.pone.0001884

Beattie, M. S., Farooqui, A. A., and Bresnahan, J. C. (2000). Review of current evidence for apoptosis after spinal cord injury. J. Neurotrauma 17, 915-925. doi: 10.1089/neu.2000.17.915

Belluzzi, E., Bisaglia, M., Lazzarini, E., Tabares, L. C., Beltramini, M., and Bubacco, L. (2012). Human SOD2 modification by dopamine quinones affects enzymatic activity by promoting its aggregation: possible implications for Parkinson's disease. PLoS One 7:e38026. doi: 10.1371/journal.pone.0038026

Blackinton, J., Kumaran, R., Van Der Brug, M. P., Ahmad, R., Olson, L., Galter, D., et al. (2009a). Post-transcriptional regulation of mRNA associated with DJ-1 in sporadic Parkinson disease. Neurosci. Lett. 452, 8-11. doi: 10.1016/j.neulet. 2008.12.053

Blackinton, J., Lakshminarasimhan, M., Thomas, K. J., Ahmad, R., Greggio, E., Raza, A. S., et al. (2009b). Formation of a stabilized cysteine sulfinic acid is critical for the mitochondrial function of the parkinsonism protein DJ-1. J. Biol. Chem. 284, 6476-6485. doi: 10.1074/jbc.M806599200

Bode, J. G., Ehlting, C., and Haussinger, D. (2012). The macrophage response towards LPS and its control through the p38(MAPK)-STAT3 axis. Cell. Signal. 24, 1185-1194. doi: 10.1016/j.cellsig.2012.01.018

Bonifati, V., Rizzu, P., Van Baren, M. J., Schaap, O., Breedveld, G. J., Krieger, E., et al. (2003). Mutations in the DJ-1 gene associated with autosomal 
recessive early-onset parkinsonism. Science 299, 256-259. doi: 10.1126/science. 1077209

Brahmachari, S., Jana, A., and Pahan, K. (2009). Sodium benzoate, a metabolite of cinnamon and a food additive, reduces microglial and astroglial inflammatory responses. J. Immunol. 183, 5917-5927. doi: 10.4049/jimmunol.0803336

Brahmachari, S., and Pahan, K. (2007). Sodium benzoate, a food additive and a metabolite of cinnamon, modifies T cells at multiple steps and inhibits adoptive transfer of experimental allergic encephalomyelitis. J. Immunol. 179, 275-283. doi: 10.4049/jimmunol.179.1.275

Byrnes, K. R., Stoica, B. A., Fricke, S., Di Giovanni, S., and Faden, A. I. (2007). Cell cycle activation contributes to post-mitotic cell death and secondary damage after spinal cord injury. Brain 130, 2977-2992. doi: 10.1093/brain/awm179

Canet-Aviles, R. M., Wilson, M. A., Miller, D. W., Ahmad, R., Mclendon, C., Bandyopadhyay, S., et al. (2004). The Parkinson's disease protein DJ-1 is neuroprotective due to cysteine-sulfinic acid-driven mitochondrial localization. Proc. Natl. Acad. Sci. U.S.A. 101, 9103-9108. doi: 10.1073/pnas.0402959101

Cao, J., Ying, M., Xie, N., Lin, G., Dong, R., Zhang, J., et al. (2014). The oxidation states of DJ-1 dictate the cell fate in response to oxidative stress triggered by 4-hpr: autophagy or apoptosis? Antioxid. Redox Signal. 21, 1443-1459. doi: 10.1089/ars.2013.5446

Casella, G. T., Bunge, M. B., and Wood, P. M. (2006). Endothelial cell loss is not a major cause of neuronal and glial cell death following contusion injury of the spinal cord. Exp. Neurol. 202, 8-20. doi: 10.1016/j.expneurol.2006.05.028

Chan, P. H. (2005). Mitochondrial dysfunction and oxidative stress as determinants of cell death/survival in stroke. Ann. N. Y. Acad. Sci. 1042, 203-209. doi: 10. 1196/annals.1338.022

Chen, L., Cagniard, B., Mathews, T., Jones, S., Koh, H. C., Ding, Y., et al. (2005). Age-dependent motor deficits and dopaminergic dysfunction in DJ-1 null mice. J. Biol. Chem. 280, 21418-21426. doi: 10.1074/jbc.M413955200

Choi, W. S., Eom, D. S., Han, B. S., Kim, W. K., Han, B. H., Choi, E. J., et al. (2004). Phosphorylation of p38 MAPK induced by oxidative stress is linked to activation of both caspase-8- and -9-mediated apoptotic pathways in dopaminergic neurons. J. Biol. Chem. 279, 20451-20460. doi: 10.1074/jbc. M311164200

Davis, S. M., and Pennypacker, K. R. (2017). Targeting antioxidant enzyme expression as a therapeutic strategy for ischemic stroke. Neurochem. Int. 107, 23-32. doi: 10.1016/j.neuint.2016.12.007

Di Segni, A., Farin, K., and Pinkas-Kramarski, R. (2006). ErbB4 activation inhibits MPP+-induced cell death in PC12-ErbB4 cells: involvement of PI3K and Erk signaling. J. Mol. Neurosci. 29, 257-267. doi: 10.1385/JMN:29:3:257

Droge, W. (2002). Free radicals in the physiological control of cell function. Physiol. Rev. 82, 47-95. doi: 10.1152/physrev.00018.2001

Dulmovits, B. M., and Herman, I. M. (2012). Microvascular remodeling and wound healing: a role for pericytes. Int. J. Biochem. Cell Biol. 44, 1800-1812. doi: 10.1016/j.biocel.2012.06.031

Fatima, G., Sharma, V. P., Das, S. K., and Mahdi, A. A. (2015). Oxidative stress and antioxidative parameters in patients with spinal cord injury: implications in the pathogenesis of disease. Spinal Cord 53, 3-6. doi: 10.1038/sc.2014.178

Figueroa, J. D., Serrano-Illan, M., Licero, J., Cordero, K., Miranda, J. D., and De Leon, M. (2016). fatty acid binding protein 5 modulates docosahexaenoic acidinduced recovery in rats undergoing spinal cord injury. J. Neurotrauma 33, 1436-1449. doi: 10.1089/neu.2015.4186

Gao, L., Xu, W., Fan, S., Li, T., Zhao, T., Ying, G., et al. (2018). MANF attenuates neuronal apoptosis and promotes behavioral recovery via Akt/MDM-2/p53 pathway after traumatic spinal cord injury in rats. Biofactors [Epub ahead of print]. doi: 10.1002/biof.1433

Ghatan, S., Larner, S., Kinoshita, Y., Hetman, M., Patel, L., Xia, Z., et al. (2000). p38 MAP kinase mediates bax translocation in nitric oxide-induced apoptosis in neurons. J. Cell Biol. 150, 335-347. doi: 10.1083/jcb.150.2.335

Goldberg, M. S., Pisani, A., Haburcak, M., Vortherms, T. A., Kitada, T., Costa, C., et al. (2005). Nigrostriatal dopaminergic deficits and hypokinesia caused by inactivation of the familial Parkinsonism-linked gene DJ-1. Neuron 45, 489496. doi: 10.1016/j.neuron.2005.01.041

Gomez-Lazaro, M., Galindo, M. F., Melero-Fernandez, De Mera, R. M., FernandezGomez, F. J., Concannon, C. G., et al. (2007). Reactive oxygen species and p38 mitogen-activated protein kinase activate Bax to induce mitochondrial cytochrome c release and apoptosis in response to malonate. Mol. Pharmacol. 71, 736-743. doi: 10.1124/mol.106.030718
Gorner, K., Holtorf, E., Waak, J., Pham, T. T., Vogt-Weisenhorn, D. M., Wurst, W., et al. (2007). Structural determinants of the C-terminal helix-kink-helix motif essential for protein stability and survival promoting activity of DJ-1. J. Biol. Chem. 282, 13680-13691. doi: 10.1074/jbc.M609821200

Gropman, A. L., Summar, M., and Leonard, J. V. (2007). Neurological implications of urea cycle disorders. J. Inherit. Metab. Dis. 30, 865-879. doi: 10.1007/s10545007-0709-5

Hod, Y., Pentyala, S. N., Whyard, T. C., and El-Maghrabi, M. R. (1999). Identification and characterization of a novel protein that regulates RNAprotein interaction. J. Cell. Biochem. 72, 435-444. doi: 10.1002/(SICI)10974644(19990301)72:3<435::AID-JCB12>3.0.CO;2-H

Hu, A. M., Li, J. J., Sun, W., Yang, D. G., Yang, M. L., Du, L. J., et al. (2015). Myelotomy reduces spinal cord edema and inhibits aquaporin-4 and aquaporin-9 expression in rats with spinal cord injury. Spinal Cord 53, 98-102. doi: $10.1038 /$ sc.2014.209

Huang, Y., Cai, G. Q., Peng, J. P., and Shen, C. (2018). Glucocorticoids induce apoptosis and matrix metalloproteinase-13 expression in chondrocytes through the NOX4/ROS/p38 MAPK pathway. J. Steroid Biochem. Mol. Biol. 181, 52-62. doi: 10.1016/j.jsbmb.2018.03.001

Hylden, J. L., and Wilcox, G. L. (1980). Intrathecal morphine in mice: a new technique. Eur. J. Pharmacol. 67, 313-316. doi: 10.1016/0014-2999(80)90515-4

Inden, M., Taira, T., Kitamura, Y., Yanagida, T., Tsuchiya, D., Takata, K., et al. (2006). PARK7 DJ-1 protects against degeneration of nigral dopaminergic neurons in Parkinson's disease rat model. Neurobiol. Dis. 24, 144-158. doi: 10.1016/j.nbd.2006.06.004

Jana, A., Modi, K. K., Roy, A., Anderson, J. A., Van Breemen, R. B., and Pahan, K. (2013). Up-regulation of neurotrophic factors by cinnamon and its metabolite sodium benzoate: therapeutic implications for neurodegenerative disorders. J. Neuroimmune Pharmacol. 8, 739-755. doi: 10.1007/s11481-013-9447-7

Jin, J., Meredith, G. E., Chen, L., Zhou, Y., Xu, J., Shie, F. S., et al. (2005). Quantitative proteomic analysis of mitochondrial proteins: relevance to Lewy body formation and Parkinson's disease. Brain Res. Mol. Brain Res. 134, 119138. doi: 10.1016/j.molbrainres.2004.10.003

Junn, E., Jang, W. H., Zhao, X., Jeong, B. S., and Mouradian, M. M. (2009). Mitochondrial localization of DJ-1 leads to enhanced neuroprotection. J. Neurosci. Res. 87, 123-129. doi: 10.1002/jnr.21831

Kahle, P. J., Waak, J., and Gasser, T. (2009). DJ-1 and prevention of oxidative stress in Parkinson's disease and other age-related disorders. Free Radic. Biol. Med. 47, 1354-1361. doi: 10.1016/j.freeradbiomed.2009.08.003

Khasnavis, S., and Pahan, K. (2012). Sodium benzoate, a metabolite of cinnamon and a food additive, upregulates neuroprotective Parkinson disease protein DJ-1 in astrocytes and neurons. J. Neuroimmune Pharmacol. 7, 424-435. doi: 10.1007/s11481-011-9286-3

Khasnavis, S., and Pahan, K. (2014). Cinnamon treatment upregulates neuroprotective proteins Parkin and DJ-1 and protects dopaminergic neurons in a mouse model of Parkinson's disease. J. Neuroimmune Pharmacol. 9, 569-581. doi: 10.1007/s11481-014-9552-2

Kim, G. W., Kondo, T., Noshita, N., and Chan, P. H. (2002). Manganese superoxide dismutase deficiency exacerbates cerebral infarction after focal cerebral ischemia/reperfusion in mice: implications for the production and role of superoxide radicals. Stroke 33, 809-815. doi: 10.1161/hs0302.103745

Kim, J. H., Choi, D. J., Jeong, H. K., Kim, J., Kim, D. W., Choi, S. Y., et al. (2013). DJ-1 facilitates the interaction between STAT1 and its phosphatase, SHP-1, in brain microglia and astrocytes: A novel anti-inflammatory function of DJ-1. Neurobiol. Dis. 60, 1-10. doi: 10.1016/j.nbd.2013.08.007

Kim, R. H., Peters, M., Jang, Y., Shi, W., Pintilie, M., Fletcher, G. C., et al. (2005a). DJ-1, a novel regulator of the tumor suppressor PTEN. Cancer Cell 7, 263-273. doi: 10.1016/j.ccr.2005.02.010

Kim, R. H., Smith, P. D., Aleyasin, H., Hayley, S., Mount, M. P., Pownall, S., et al. (2005b). Hypersensitivity of DJ-1-deficient mice to 1-methyl-4-phenyl-1,2,3,6tetrahydropyrindine (MPTP) and oxidative stress. Proc. Natl. Acad. Sci. U.S.A. 102, 5215-5220. doi: 10.1073/pnas.0501282102

Kim, W., Kim, D. W., Jeong, H. J., Yoo, D. Y., Jung, H. Y., Nam, S. M., et al. (2014). Tat-DJ-1 protects neurons from ischemic damage in the ventral horn of rabbit spinal cord via increasing antioxidant levels. Neurochem. Res. 39, 187-193. doi: 10.1007/s11064-013-1205-y

Kinumi, T., Kimata, J., Taira, T., Ariga, H., and Niki, E. (2004). Cysteine-106 of DJ-1 is the most sensitive cysteine residue to hydrogen peroxide-mediated 
oxidation in vivo in human umbilical vein endothelial cells. Biochem. Biophys. Res. Commun. 317, 722-728. doi: 10.1016/j.bbrc.2004.03.110

Kjell, J., Olson, L., and Abrams, M. B. (2016). Improved recovery from spinal cord injury in rats with chronic parvovirus serotype-1a infection. Spinal Cord 54, 517-520. doi: 10.1038/sc.2015.208

Ko, H. R., Kwon, I. S., Hwang, I., Jin, E. J., Shin, J. H., Brennan-Minnella, A. M., et al. (2016). Akt1-Inhibitor of DNA binding2 is essential for growth cone formation and axon growth and promotes central nervous system axon regeneration. eLife 5:e20799. doi: 10.7554/eLife.20799

Krebiehl, G., Ruckerbauer, S., Burbulla, L. F., Kieper, N., Maurer, B., Waak, J., et al. (2010). Reduced basal autophagy and impaired mitochondrial dynamics due to loss of Parkinson's disease-associated protein DJ-1. PLoS One 5:e9367. doi: 10.1371/journal.pone.0009367

Kubota, K., and Ishizaki, T. (1991). Dose-dependent pharmacokinetics of benzoic acid following oral administration of sodium benzoate to humans. Eur. J. Clin. Pharmacol. 41, 363-368. doi: 10.1007/BF00314969

Kundu, M., Mondal, S., Roy, A., Martinson, J. L., and Pahan, K. (2016). Sodium benzoate, a food additive and a metabolite of cinnamon, enriches regulatory T cells via STAT6-mediated upregulation of TGF-beta. J. Immunol. 197, 30993110. doi: 10.4049/jimmunol.1501628

Lam, T., Chen, Z., Sayed-Ahmed, M. M., Krassioukov, A., and Al-Yahya, A. A. (2013). Potential role of oxidative stress on the prescription of rehabilitation interventions in spinal cord injury. Spinal Cord 51, 656-662. doi: 10.1038/sc. 2013.71

Lee, J. Y., Kim, H. S., Choi, H. Y., Oh, T. H., Ju, B. G., and Yune, T. Y. (2012). Valproic acid attenuates blood-spinal cord barrier disruption by inhibiting matrix metalloprotease- 9 activity and improves functional recovery after spinal cord injury. J. Neurochem. 121, 818-829. doi: 10.1111/j.1471-4159.2012.07731.x

Li, X. Q., Lv, H. W., Tan, W. F., Fang, B., Wang, H., and Ma, H. (2014). Role of the TLR4 pathway in blood-spinal cord barrier dysfunction during the bimodal stage after ischemia/reperfusion injury in rats. J. Neuroinflammation 11:62. doi: 10.1186/1742-2094-11-62

Liang, W., Han, Q., Jin, W., Xiao, Z., Huang, J., Ni, H., et al. (2010). The promotion of neurological recovery in the rat spinal cord crushed injury model by collagenbinding BDNF. Biomaterials 31, 8634-8641. doi: 10.1016/j.biomaterials.2010. 07.084

Liu, X. Z., Xu, X. M., Hu, R., Du, C., Zhang, S. X., Mcdonald, J. W., et al. (1997). Neuronal and glial apoptosis after traumatic spinal cord injury. J. Neurosci. 17, 5395-5406. doi: 10.1523/JNEUROSCI.17-14-05395.1997

Malhotra, D., Thimmulappa, R., Navas-Acien, A., Sandford, A., Elliott, M., Singh, A., et al. (2008). Decline in NRF2-regulated antioxidants in chronic obstructive pulmonary disease lungs due to loss of its positive regulator, DJ-1. Am. J. Respir. Crit. Care Med. 178, 592-604. doi: 10.1164/rccm.200803-380OC

Manning, B. D., and Toker, A. (2017). AKT/PKB signaling: navigating the network. Cell 169, 381-405. doi: 10.1016/j.cell.2017.04.001

Martinat, C., Shendelman, S., Jonason, A., Leete, T., Beal, M. F., Yang, L., et al. (2004). Sensitivity to oxidative stress in DJ-1-deficient dopamine neurons: an ES- derived cell model of primary Parkinsonism. PLoS Biol. 2:e327. doi: 10. 1371/journal.pbio.0020327

Mattson, M. P. (2000). Apoptosis in neurodegenerative disorders. Nat. Rev. Mol. Cell Biol. 1, 120-129. doi: 10.1038/35040009

Mehta, S. L., Lin, Y., Chen, W., Yu, F., Cao, L., He, Q., et al. (2011). Manganese superoxide dismutase deficiency exacerbates ischemic brain damage under hyperglycemic conditions by altering autophagy. Transl. Stroke Res. 2, 42-50. doi: 10.1007/s12975-010-0027-3

Misel, M. L., Gish, R. G., Patton, H., and Mendler, M. (2013). Sodium benzoate for treatment of hepatic encephalopathy. Gastroenterol. Hepatol. 9, 219-227.

Mita, Y., Kataoka, Y., Saito, Y., Kashi, T., Hayashi, K., Iwasaki, A., et al. (2018). Distribution of oxidized DJ-1 in Parkinson's disease-related sites in the brain and in the peripheral tissues: effects of aging and neurotoxin. Sci. Rep. 8:12056. doi: 10.1038/s41598-018-30561-z

Mitsumoto, A., Nakagawa, Y., Takeuchi, A., Okawa, K., Iwamatsu, A., and Takanezawa, Y. (2001). Oxidized forms of peroxiredoxins and DJ-1 on twodimensional gels increased in response to sublethal levels of paraquat. Free Radic. Res. 35, 301-310. doi: 10.1080/10715760100300831

Mizuno, Y., Mochizuki, H., Sugita, Y., and Goto, K. (1998). Apoptosis in neurodegenerative disorders. Intern. Med. 37, 192-193. doi: 10.2169/ internalmedicine.37.192
Mo, J. S., Jung, J., Yoon, J. H., Hong, J. A., Kim, M. Y., Ann, E. J., et al. (2010). DJ-1 modulates the p38 mitogen-activated protein kinase pathway through physical interaction with apoptosis signal-regulating kinase 1. J. Cell. Biochem. 110, 229-237. doi: 10.1002/jcb.22530

Modi, K. K., Roy, A., Brahmachari, S., Rangasamy, S. B., and Pahan, K. (2015). Cinnamon and its metabolite sodium benzoate attenuate the activation of p21rac and protect memory and learning in an animal model of Alzheimer's disease. PLoS One 10:e0130398. doi: 10.1371/journal.pone.0130398

Nagakubo, D., Taira, T., Kitaura, H., Ikeda, M., Tamai, K., Iguchi-Ariga, S. M., et al. (1997). DJ-1, a novel oncogene which transforms mouse NIH3T3 cells in cooperation with ras. Biochem. Biophys. Res. Commun. 231, 509-513. doi: 10.1006/bbrc. 1997.6132

Nair, B. (2001). Final report on the safety assessment of Benzyl alcohol, benzoic acid, and sodium benzoate. Int. J. Toxicol. 20(Suppl. 3), 23-50. doi: 10.1080/ 10915810152630729

Nesic, O., Lee, J., Ye, Z., Unabia, G. C., Rafati, D., Hulsebosch, C. E., et al. (2006). Acute and chronic changes in aquaporin 4 expression after spinal cord injury. Neuroscience 143, 779-792. doi: 10.1016/j.neuroscience.2006.08.079

Niu, C., and Yip, H. K. (2011). Neuroprotective signaling mechanisms of telomerase are regulated by brain-derived neurotrophic factor in rat spinal cord motor neurons. J. Neuropathol. Exp. Neurol. 70, 634-652. doi: 10.1097/NEN. 0b013e318222b97b

Okada, M., Matsumoto, K., Niki, T., Taira, T., Iguchi-Ariga, S. M., and Ariga, H. (2002). DJ-1, a target protein for an endocrine disrupter, participates in the fertilization in mice. Biol. Pharm. Bull. 25, 853-856. doi: 10.1248/bpb.25.853

Ooe, H., Taira, T., Iguchi-Ariga, S. M., and Ariga, H. (2005). Induction of reactive oxygen species by bisphenol A and abrogation of bisphenol A-induced cell injury by DJ-1. Toxicol. Sci. 88, 114-126. doi: 10.1093/toxsci/kfi278

Pantcheva, P., Elias, M., Duncan, K., Borlongan, C. V., Tajiri, N., and Kaneko, Y. (2014). The role of DJ-1 in the oxidative stress cell death cascade after stroke. Neural Regen. Res. 9, 1430-1433. doi: 10.4103/1673-5374.139458

Qu, D., Rashidian, J., Mount, M. P., Aleyasin, H., Parsanejad, M., Lira, A., et al. (2007). Role of Cdk5-mediated phosphorylation of Prx2 in MPTP toxicity and Parkinson's disease. Neuron 55, 37-52. doi: 10.1016/j.neuron.2007.05.033

Rivlin, A. S., and Tator, C. H. (1977). Objective clinical assessment of motor function after experimental spinal cord injury in the rat. J. Neurosurg. 47, 577-581. doi: 10.3171/jns.1977.47.4.0577

Saha, S., Sadhukhan, P., Sinha, K., Agarwal, N., and Sil, P. C. (2016). Mangiferin attenuates oxidative stress induced renal cell damage through activation of PI3K induced Akt and Nrf-2 mediated signaling pathways. Biochem. Biophys. Rep. 5, 313-327. doi: 10.1016/j.bbrep.2016.01.011

Saito, Y. (2014). Oxidized DJ-1 as a possible biomarker of Parkinson's disease. J. Clin. Biochem. Nutr. 54, 138-144. doi: 10.3164/jcbn.13-108

Saito, Y., Miyasaka, T., Hatsuta, H., Takahashi-Niki, K., Hayashi, K., Mita, Y., et al. (2014). Immunostaining of oxidized DJ-1 in human and mouse brains. J. Neuropathol. Exp. Neurol. 73, 714-728. doi: 10.1097/NEN.0000000000000087

Sakurai, M., Kawamura, T., Nishimura, H., Suzuki, H., Tezuka, F., and Abe, K. (2009). Induction of Parkinson disease-related proteins in motor neurons after transient spinal cord ischemia in rabbits. J. Cereb. Blood Flow Metab. 29, 752-758. doi: 10.1038/jcbfm.2008.167

Scaglia, F., Carter, S., O’brien, W. E., and Lee, B. (2004). Effect of alternative pathway therapy on branched chain amino acid metabolism in urea cycle disorder patients. Mol. Genet. Metab. 81(Suppl. 1), S79-S85. doi: 10.1016/j. ymgme.2003.11.017

Sekito, A., Koide-Yoshida, S., Niki, T., Taira, T., Iguchi-Ariga, S. M., and Ariga, H. (2006). DJ-1 interacts with HIPK1 and affects H2O2-induced cell death. Free Radic. Res. 40, 155-165. doi: 10.1080/10715760500456847

Seo, J. Y., Kim, Y. H., Kim, J. W., Kim, S. I., and Ha, K. Y. (2015). Effects of therapeutic hypothermia on apoptosis and autophagy after spinal cord injury in rats. Spine 40, 883-890. doi: 10.1097/BRS.0000000000000845

Sharma, H. S. (2011). Early microvascular reactions and blood-spinal cord barrier disruption are instrumental in pathophysiology of spinal cord injury and repair: novel therapeutic strategies including nanowired drug delivery to enhance neuroprotection. J. Neural Transm. 118, 155-176. doi: 10.1007/s00702-0100514-4

Shendelman, S., Jonason, A., Martinat, C., Leete, T., and Abeliovich, A. (2004). DJ-1 is a redox-dependent molecular chaperone that inhibits alpha-synuclein aggregate formation. PLoS Biol. 2:e362. doi: 10.1371/journal.pbio.0020362 
Shimizu, Y., Lambert, J. P., Nicholson, C. K., Kim, J. J., Wolfson, D. W., Cho, H. C., et al. (2016). DJ-1 protects the heart against ischemia-reperfusion injury by regulating mitochondrial fission. J. Mol. Cell. Cardiol. 97, 56-66. doi: 10.1016/j. yjmcc.2016.04.008

Sugawara, T., and Chan, P. H. (2003). Reactive oxygen radicals and pathogenesis of neuronal death after cerebral ischemia. Antioxid. Redox Signal. 5, 597-607. doi: $10.1089 / 152308603770310266$

Sushma, S., Dasarathy, S., Tandon, R. K., Jain, S., Gupta, S., and Bhist, M. S. (1992). Sodium benzoate in the treatment of acute hepatic encephalopathy: a double-blind randomized trial. Hepatology 16, 138-144. doi: 10.1002/hep. 1840160123

Taira, T., Saito, Y., Niki, T., Iguchi-Ariga, S. M., Takahashi, K., and Ariga, H. (2004). DJ-1 has a role in antioxidative stress to prevent cell death. EMBO Rep. 5, 213-218. doi: 10.1038/sj.embor.7400074

Takahashi-Niki, K., Niki, T., Iguchi-Ariga, S. M. M., and Ariga, H. (2017). Transcriptional regulation of DJ-1. Adv. Exp. Med. Biol. 1037, 89-95. doi: 10.1007/978-981-10-6583-5_7

Toth, B. (1984). Lack of tumorigenicity of sodium benzoate in mice. Fundam. Appl. Toxicol. 4, 494-496. doi: 10.1016/0272-0590(84)90208-2

Valko, M., Leibfritz, D., Moncol, J., Cronin, M. T., Mazur, M., and Telser, J. (2007). Free radicals and antioxidants in normal physiological functions and human disease. Int. J. Biochem. Cell Biol. 39, 44-84. doi: 10.1016/j.biocel.2006. 07.001

Van Der Brug, M. P., Blackinton, J., Chandran, J., Hao, L. Y., Lal, A., MazanMamczarz, K., et al. (2008). RNA binding activity of the recessive parkinsonism protein DJ-1 supports involvement in multiple cellular pathways. Proc. Natl. Acad. Sci. U.S.A. 105, 10244-10249. doi: 10.1073/pnas.0708518105

Van Duijn, C. M., Dekker, M. C., Bonifati, V., Galjaard, R. J., HouwingDuistermaat, J. J., Snijders, P. J., et al. (2001). Park7, a novel locus for autosomal recessive early-onset parkinsonism, on chromosome 1p36. Am. J. Hum. Genet. 69, 629-634. doi: 10.1086/322996

Wang, B., Qin, H., Wang, Y., Chen, W., Luo, J., Zhu, X., et al. (2014). Effect of DJ-1 overexpression on the proliferation, apoptosis, invasion and migration of laryngeal squamous cell carcinoma SNU-46 cells through PI3K/AKT/mTOR. Oncol. Rep. 32, 1108-1116. doi: 10.3892/or.2014.3286

Wang, J. Y., Shum, A. Y., Ho, Y. J., and Wang, J. Y. (2003). Oxidative neurotoxicity in rat cerebral cortex neurons: synergistic effects of $\mathrm{H} 2 \mathrm{O} 2$ and $\mathrm{NO}$ on apoptosis involving activation of $\mathrm{p} 38$ mitogen-activated protein kinase and caspase-3. J. Neurosci. Res. 72, 508-519. doi: 10.1002/jnr.10597

Wang, S., Liu, Y., Wu, C., Zhao, W., Zhang, J., Bao, G., et al. (2017). The expression of IGFBP6 after spinal cord injury: implications for neuronal apoptosis. Neurochem. Res. 42, 455-467. doi: 10.1007/s11064-016-2092-9

Wang, Y., Sun, Y., Zhao, X., Yuan, R., Jiang, H., and Pu, X. (2018). Downregulation of DJ-1 Fails to Protect Mitochondrial Complex I Subunit NDUFS3 in the Testes and Contributes to the Asthenozoospermia. Mediators Inflamm. 2018:6136075. doi: 10.1155/2018/6136075

Wang, Y. F., Fan, Z. K., Cao, Y., Yu, D. S., Zhang, Y. Q., and Wang, Y. S. (2011). 2-Methoxyestradiol inhibits the up-regulation of AQP4 and AQP1 expression after spinal cord injury. Brain Res. 1370, 220-226. doi: 10.1016/j.brainres.2010. 11.016

Wiener, H. W., Perry, R. T., Chen, Z., Harrell, L. E., and Go, R. C. (2007). A polymorphism in SOD2 is associated with development of Alzheimer's disease. Genes Brain Behav. 6, 770-775. doi: 10.1111/j.1601-183X.2007.00308.x

Williams, R. E., and Lock, E. A. (2005). Sodium benzoate attenuates D-serine induced nephrotoxicity in the rat. Toxicology 207, 35-48. doi: 10.1016/j.tox. 2004.08.008

Wu, Q., Jing, Y., Yuan, X., Zhang, X., Li, B., Liu, M., et al. (2014). Melatonin treatment protects against acute spinal cord injury-induced disruption of blood spinal cord barrier in mice. J. Mol. Neurosci. 54, 714-722. doi: 10.1007/s12031014-0430-4

Xu, J., Zhong, N., Wang, H., Elias, J. E., Kim, C. Y., Woldman, I., et al. (2005). The Parkinson's disease-associated DJ-1 protein is a transcriptional co-activator that protects against neuronal apoptosis. Hum. Mol. Genet. 14, 1231-1241. doi: $10.1093 / \mathrm{hmg} / \mathrm{ddi1} 34$
Yamagishi, Y., Saigoh, K., Saito, Y., Ogawa, I., Mitsui, Y., Hamada, Y., et al. (2018). Diagnosis of Parkinson's disease and the level of oxidized DJ-1 protein. Neurosci. Res. 128, 58-62. doi: 10.1016/j.neures.2017.06.008

Yan, F., Cao, S., Li, J., Dixon, B., Yu, X., Chen, J., et al. (2017). Pharmacological inhibition of PERK attenuates early brain injury after subarachnoid hemorrhage in rats through the activation of Akt. Mol. Neurobiol. 54, 1808-1817. doi: 10.1007/s12035-016-9790-9

Yanagida, T., Tsushima, J., Kitamura, Y., Yanagisawa, D., Takata, K., Shibaike, T., et al. (2009). Oxidative stress induction of DJ-1 protein in reactive astrocytes scavenges free radicals and reduces cell injury. Oxid. Med. Cell. Longev. 2, 36-42. doi: 10.4161/oxim.2.1.7985

Yanagisawa, D., Kitamura, Y., Inden, M., Takata, K., Taniguchi, T., Morikawa, S., et al. (2008). DJ-1 protects against neurodegeneration caused by focal cerebral ischemia and reperfusion in rats. J. Cereb. Blood Flow Metab. 28, 563-578. doi: $10.1038 /$ sj.jcbfm. 9600553

Yang, Y., Gehrke, S., Haque, M. E., Imai, Y., Kosek, J., Yang, L., et al. (2005). Inactivation of Drosophila DJ-1 leads to impairments of oxidative stress response and phosphatidylinositol 3-kinase/Akt signaling. Proc. Natl. Acad. Sci. U.S.A. 102, 13670-13675. doi: 10.1073/pnas.0504610102

Yokota, T., Sugawara, K., Ito, K., Takahashi, R., Ariga, H., and Mizusawa, H. (2003). Down regulation of DJ-1 enhances cell death by oxidative stress, ER stress, and proteasome inhibition. Biochem. Biophys. Res. Commun. 312, 1342-1348. doi: 10.1016/j.bbrc.2003.11.056

Yoshida, K., Sato, Y., Yoshiike, M., Nozawa, S., Ariga, H., and Iwamoto, T. (2003). Immunocytochemical localization of DJ-1 in human male reproductive tissue. Mol. Reprod. Dev. 66, 391-397. doi: 10.1002/mrd.10360

Zhang, L., Shimoji, M., Thomas, B., Moore, D. J., Yu, S. W., Marupudi, N. I., et al. (2005). Mitochondrial localization of the Parkinson's disease related protein DJ-1: implications for pathogenesis. Hum. Mol. Genet. 14, 2063-2073. doi: 10.1093/hmg/ddi211

Zhang, W., Cheng, L., Hou, Y., Si, M., Zhao, Y. P., and Nie, L. (2015). Plumbagin protects against spinal cord injury-induced oxidative stress and inflammation in Wistar rats through Nrf-2 upregulation. Drug Res. 65, 495-499.

Zhang, Y., Gong, X. G., Wang, Z. Z., Sun, H. M., Guo, Z. Y., Hu, J. H., et al. (2016). Overexpression of DJ-1/PARK7, the Parkinson's disease-related protein, improves mitochondrial function via Akt phosphorylation on threonine 308 in dopaminergic neuron-like cells. Eur. J. Neurosci. 43, 1379-1388. doi: 10.1111/ ejn. 13216

Zhong, N., Kim, C. Y., Rizzu, P., Geula, C., Porter, D. R., Pothos, E. N., et al. (2006). DJ-1 transcriptionally up-regulates the human tyrosine hydroxylase by inhibiting the sumoylation of pyrimidine tract-binding protein-associated splicing factor. J. Biol. Chem. 281, 20940-20948. doi: 10.1074/jbc.M601935200

Zhong, N., and Xu, J. (2008). Synergistic activation of the human MnSOD promoter by DJ-1 and PGC-1alpha: regulation by SUMOylation and oxidation. Hum. Mol. Genet. 17, 3357-3367. doi: 10.1093/hmg/ddn230

Zhou, W., Zhu, M., Wilson, M. A., Petsko, G. A., and Fink, A. L. (2006). The oxidation state of DJ-1 regulates its chaperone activity toward alpha-synuclein. J. Mol. Biol. 356, 1036-1048. doi: 10.1016/j.jmb.2005.12.030

Zhou, Y., Wu, Y., Liu, Y., He, Z., Zou, S., Wang, Q., et al. (2017). The crosstalk between autophagy and endoplasmic reticulum stress in blood-spinal cord barrier disruption after spinal cord injury. Oncotarget 8, 1688-1702. doi: 10. 18632/oncotarget.13777

Conflict of Interest Statement: The authors declare that the research was conducted in the absence of any commercial or financial relationships that could be construed as a potential conflict of interest.

Copyright $\odot 2019$ Gao, Zhang, Xu, Li, Ying, Qin, Li, Zheng, Zhao, Yan, Zhu and Chen. This is an open-access article distributed under the terms of the Creative Commons Attribution License (CC BY). The use, distribution or reproduction in other forums is permitted, provided the original author(s) and the copyright owner(s) are credited and that the original publication in this journal is cited, in accordance with accepted academic practice. No use, distribution or reproduction is permitted which does not comply with these terms. 